\title{
Évolution des forêts québécoises au regard des habitats fauniques : analyse des grandes tendances sur trois décennies ${ }^{1}$
}

\author{
par Michel Crête ${ }^{2}$ et Lothar Marzell²
}

\begin{abstract}
RÉSUMÉ
Comme l'exploitation forestière s'est intensifiée entre les années 1970 et 1990, nous avons vérifié la prédiction que trois attributs des forêts, vraisemblablement essentiels à certains éléments de la faune québécoise, aient régressé durant cette période : les forêts âgées (> 100 ans), le bois mort et la diversité des arbres et arbustes. Nous avons utilisé un réseau de près de 7000 placettes permanentes, inventoriées à au moins trois reprises, pour déterminer la tendance suivie par ces variables au cours des trois dernières décennies du $20^{\mathrm{e}}$ siècle. Nous avons stratifié notre analyse en fonction des six domaines bioclimatiques qui couvrent la moitié sud du Québec et où a cours l'aménagement forestier, soit de l'érablière à caryer à la pessière à mousses. La proportion de forêts âgées n’a régressé clairement que dans la partie ouest de la sapinière et de l'érablière à bouleau jaune. Toutefois, les peuplements composés d'arbres âgés s'avéraient déjà très rares durant les années 1970 partout, sauf dans la pessière à mousses où ils ont vraisemblablement légèrement gagné en importance durant la période d'étude. Les chicots ont eu tendance à se raréfier uniquement dans la partie ouest de la sapinière et de l'érablière à bouleau jaune alors qu'ailleurs ils ont augmenté en nombre, parfois substantiellement, à cause de l'épidémie de la tordeuse des bourgeons de l'épinette qui a affecté tout le Québec entre 1975 et 1990. Les résultats suggèrent que la diversité des arbres ait été appauvrie dans les deux domaines bioclimatiques les plus au sud; la même tendance existait pour les gaules, notamment à cause du broutage intense du cerf de Virginie. Dans la forêt boréale, l'épidémie de la tordeuse des bourgeons de l'épinette a favorisé une plus grande diversité de gaules durant les années 1980 et 1990. Notre analyse indique qu'il faut : 1) rapidement exclure de l'aménagement forestier des échantillons représentatifs de forêts âgées dans tous les domaines bioclimatiques, 2) déterminer si des espèces ligneuses se sont raréfiées dans les forêts du sud du Québec, 3) déterminer quels éléments de la faune québécoise sont tributaires des forêts âgées, des espèces d'arbre rares ou d'arbres vétérans et 4) continuer à suivre l'évolution de la quantité de bois mort présent dans les forêts du Québec.
\end{abstract}

Mots-clés : aménagement, conservation, exploitation, faune, forêt, gestion, Québec

\section{ABSTRACT}

As forest management intensified between the 1970s and the 1990s, we tested the prediction that three forest attributes, likely essential for some wildlife species, became rarer during this time interval: old (> 100 years) stands, dead wood and woody species diversity. We used a network of about 7000 permanent plots, surveyed at least three times, for determining trends followed by these variables during the last three decades of the $20^{\text {th }}$ century. We stratified our analysis according to the six vegetation domains of the southern half of Québec where forest management occurs, i.e., from the sugar maplebitternut to the spruce-moss domain. The proportion of old stands clearly diminished only in the western part of the sugar maple- and balsam fir-yellow birch domains. However, stands composed of old trees were already very scarce during the 1970s everywhere except in the spruce-moss domain where they could have increased in importance during the study period. Snags tended to become rarer only in the western part of sugar maple- and balsam fir-yellow birch domains whereas their abundance increased elsewhere, sometimes substantially, because of the spruce budworm epidemic that affected Québec between 1975 and 1990. Results suggest that tree diversity was impoverished in the two southernmost forest domains; the same tendency existed also for saplings, particularly because of intense browsing by white-tailed deer. In the boreal forest, the spruce budworm epidemic favoured sapling diversity during the 1980s and 1990s. Our analysis indicates that we must: 1) quickly exclude some typical old stands from forest management in all vegetation domains; 2) determine if some woody species became rarer in forest stands of southern Québec; 3) identify which elements of the forest fauna depend on old stands, rare tree species and senescent trees, and 4) continue to monitor the trend of dead wood present in Québec forests.

Key words: conservation, forest, harvest, management, Québec, wildlife

\footnotetext{
${ }^{1}$ Présenté au 5th North American Forest Ecology Workshop, Gatineau, Quebec, June 2005.

${ }^{2}$ Ministère des Ressources naturelles et de la Faune, Direction du développement de la faune, 930, Chemin Sainte-Foy, Québec G1S 2L4. Courriel : lothar.marzell@fapaq.gouv.qc.ca
} 


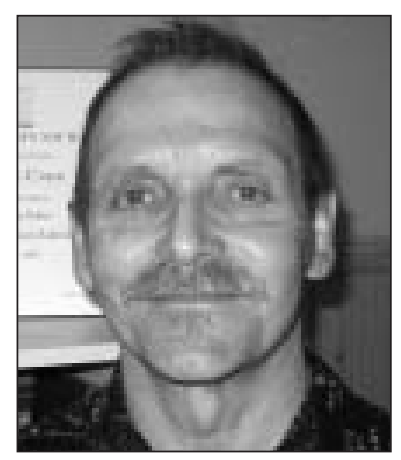

Michel Crête

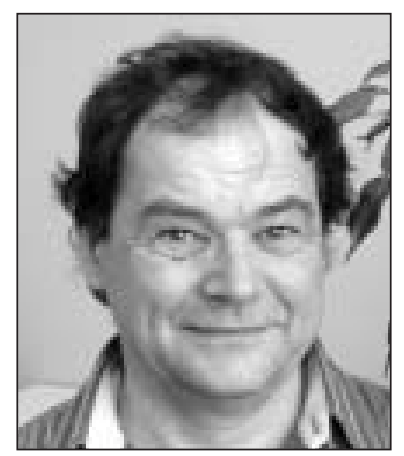

Lothar Marzell

\section{Introduction}

La forêt a joué, depuis toujours, un rôle central dans l'économie québécoise. En effet, les premiers occupants du territoire tiraient de la forêt des aliments, des vêtements et de l'énergie pour assurer leur subsistance (Anonyme 1972). Plus tard, la colonisation de la vallée du Saint-Laurent par les Européens a progressivement conduit à une intensification de l'exploitation des ressources forestières. Par exemple, la récolte du bois, notamment pour l'industrie navale, conduisit à une raréfaction du chêne (Quercus sp.) et du pin (Pinus sp.) au début du XIX ${ }^{\mathrm{e}}$ siècle dans le Haut Saint-Laurent (Simard et Bouchard 1996). Au $20^{\mathrm{e}}$ siècle, la croissance démographique et l'industrialisation accélérèrent la pression exercée sur la forêt, qui culmina dans les années 1990. Ainsi, on récoltait annuellement environ $30 \cdot 10^{6} \mathrm{~m}^{3}$ de bois au début des années 1980 (Parent 1991) et $44 \cdot 10^{6} \mathrm{~m}^{3}$ en 1999 (Parent et Fortin 2003); au cours des dernières années, on a récolté plus de $89 \%$ du potentiel annuel estimé pour les essences résineuses de toutes les forêts québécoises (Parent et Fortin 2003).

Dans les forêts équiennes, l'aménagement forestier classique, utilisé jusqu'à maintenant au Québec, vise la normalisation de l'âge des peuplements forestiers, de sorte que toutes les classes d'âge occupent une importance égale; en forêt inéquienne, on tente de régulariser le nombre de tiges par classe de diamètre à hauteur de poitrine (DHP) (Barette et al. 1996). Ainsi, théoriquement, les peuplements ne dépassent pas l'âge de la maturité dans les forêts équiennes aménagées alors qu'aucune tige n'atteint la sénescence dans les forêts inéquiennes. Les forêts aménagées se distinguent donc grandement des forêts subissant un régime de perturbations naturelles qui renferment des peuplements et des arbres très âgés. Par exemple, $37 \%$ des peuplements excèdent l'âge de 100 ans sous un cycle de feu de 100 ans, contre aucun pour des forêts de même nature qui seraient aménagées avec une période de rotation identique de 100 ans (Bergeron 2004).

L'exploitation forestière conduit inéluctablement à une raréfaction du bois mort dans les forêts aménagées puisque la coupe se substitue en grande partie à la mortalité naturelle. Par exemple, les chicots et les débris ligneux au sol occupent entre 14 et $20 \mathrm{~m}^{3} \cdot \mathrm{ha}^{-1}$ dans les forêts russes aménagées contre 40 à $51 \mathrm{~m}^{3} \cdot \mathrm{ha}^{-1}$ dans les forêts naturelles (Krankina et al. 2002). Dans les sapinières de l'est du Québec, l'aménagement forestier a produit des effets semblables : 40 à $63 \mathrm{~m}^{3} \cdot \mathrm{ha}^{-1}$ de débris ligneux dans les vieilles forêts naturelles contre $14 \mathrm{~m}^{3} \cdot \mathrm{ha}^{-1}$ dans les sapinières de seconde venue (Desponts et al. 2004).

Plusieurs traitements sylvicoles utilisés au Québec peuvent modifier la composition des peuplements forestiers à diverses étapes de leur croissance, telles que la plantation, l'éclaircie précommerciale ou l'éclaircie commerciale (Anonyme 2003). Ce genre de traitements sylvicoles a touché plus de $2000 \mathrm{~km}^{2}$ en 2002-2003, soit 0,5\% du territoire forestier productif (Parent et Fortin 2003). Le marché dicte le choix des espèces privilégiées; la demande actuelle est plus forte pour les coniferes que les feuillus (Parent et Fortin 2003). De même, la coupe stimule la prolifération de certaines espèces végétales, comme par exemple le peuplier faux-tremble (Populus tremuloides), de sorte que plusieurs craignent une augmentation de la proportion des feuillus par rapport à celle issue de perturbations naturelles (enfeuillement) (Grondin et al. 2003). La suppression du feu et son remplacement par la coupe peuvent également modifier la composition des peuplements forestiers (Friedman et Reich 2005).

On a établi, au début des années 1970, un réseau de plusieurs milliers de placettes-échantillons permanentes réparties aléatoirement dans l'ensemble des forêts aménageables du Québec, soit plus de $500000 \mathrm{~km}^{2}$ (Anonyme 2001a). Dans chaque placette, on a inventorié, à intervalles d'environ 10 ans, les arbres et les gaules qui s'y trouvaient, de sorte qu'on peut maintenant étudier les tendances de la forêt québécoise sur un horizon de trois décennies. Comme la faune québécoise compte environ 30000 espèces, dont 25 000 sont des insectes méconnus (Anonyme 2001b), sa conservation doit s'appuyer sur le concept du filtre brut (Hunter 1999). Aussi, les gestionnaires de la faune désirent maintenir tous les types d'écosystèmes forestiers et toutes les espèces ligneuses afin de conserver la biodiversité animale dans les forêts aménagées. Trois préoccupations émergent en ce qui concerne l'habitat de la faune forestière : la raréfaction des peuplements âgées, la diminution du bois mort et la perte de diversité des plantes ligneuses au niveau des peuplements et des tiges individuelles. Notre analyse vise à décrire, à grands traits, l'évolution des forêts québécoises au cours des 30 dernières années, dans la perspective de la conservation de la faune forestière. Nous émettons l'hypothèse que l'aménagement forestier a imprimé une marque dominante sur l'évolution des forêts québécoises entre les années 1970 et 1990. Nous prédisons que les forêts âgées et le bois mort se sont raréfiés pendant cette période, alors que la flore ligneuse s'est appauvrie.

\section{Méthode}

Nous avons utilisé les 11 sous-domaines bioclimatiques du Québec méridional (761 $000 \mathrm{~km}^{2}$ ) comme base de stratification pour notre analyse (Saucier et al. 1998; Fig. 1). L'aménagement forestier avait cours dans tous les domaines bioclimatiques, à l'exception d'une fraction de la pessière à mousses trop peu productive pour l'exploitation. Nous avons analysé les données des placettes-permanentes du réseau de base 1 qui, à compter du début des années 1970, ont été réparties au hasard dans tous les domaines bioclimatiques, à une densité de 1 placette $/ 259 \mathrm{~km}^{2}$ dans la pessière, de 1 placette $/ 104 \mathrm{~km}^{2}$ dans les sapinières et de 1 placette $/ 26 \mathrm{~km}^{2}$ dans les érablières. Au moment de l'analyse, $23 \%$ et $18 \%$ des 


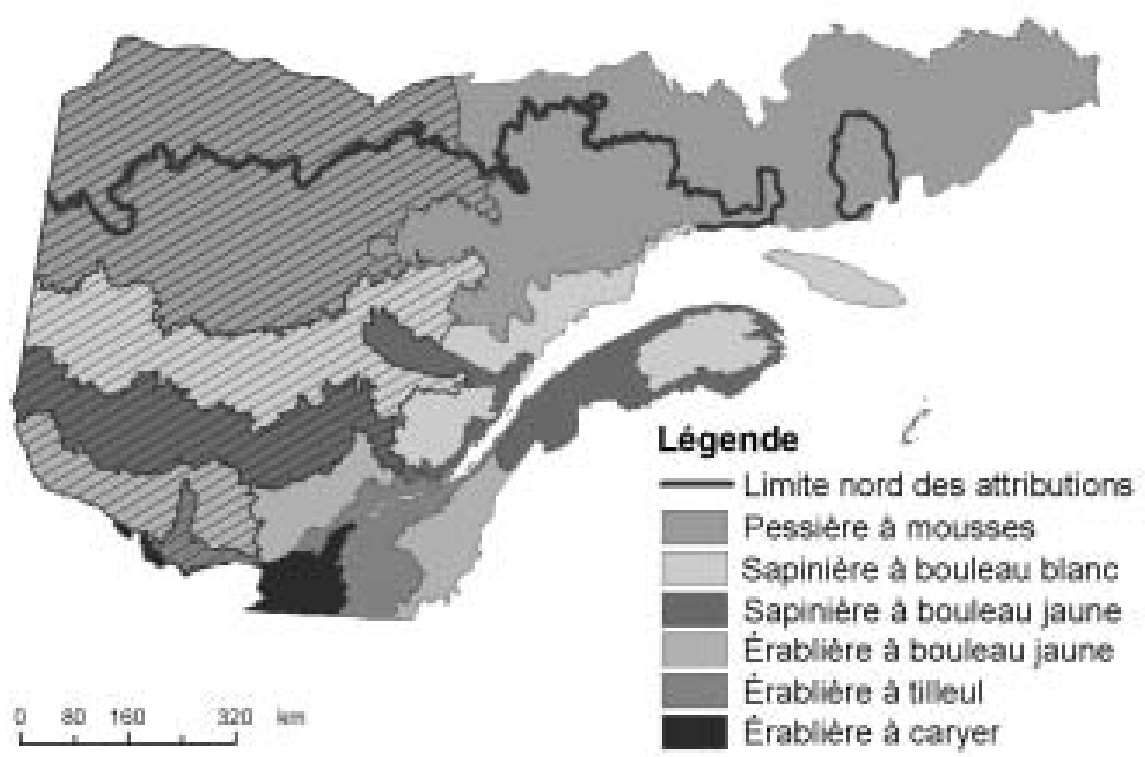

Fig. 1. Répartition des six domaines bioclimatiques couvrant le sud du Québec (d'après Saucier et al. 1998). À l'exception du domaine de l'érablière à caryer, ceux-ci se subdivisent en partie est et ouest (zone hachurée). Du sud au nord, les domaines bioclimatiques couvrent respectivement $15,30,66,99,139$ et $430 \cdot 10^{3} \mathrm{~km}^{2}$.

placettes de la pessière à mousses de l'est et de l'ouest, respectivement, se retrouvaient un peu à l'extérieur de la limite des forêts aménagées (Fig. 1). Les placettes ont été réparties par grappe de deux, ce qui correspond à une journée de travail pour une équipe de techniciens. La localisation des placettes est mesurée précisément (Anonyme 2001a) de sorte qu'elles sont marquées à nouveau si une perturbation naturelle (p. ex.. le feu) ou humaine (p. ex. la coupe) détruit les marques initiales. Quand une perturbation permanente (p. ex. une route) détruit une placette d'une paire $(<1 \%$ des cas), elle est remplacée au hasard, en conservant la même distance de la placette intacte de la paire.

Les placettes servant au dénombrement des arbres marchands $(\mathrm{DHP}>9 \mathrm{~cm})$ sont circulaires et couvrent 400 $\mathrm{m}^{2}$; tous les arbres portent un numéro pour une identification individuelle lors du remesurage. Les gaules (DHP 1-9 cm) sont dénombrées dans des placettes circulaires de $40 \mathrm{~m}^{2}$, dont le centre se superpose à celui des placettes pour les arbres; les gaules ne portent pas de numéro. Les placettes furent inventoriées à un intervalle de \pm 10 ans depuis leur mise en place. Nous avons regroupé les résultats par décennie pour toutes les analyses, soit les années 1970, 1980 et 1990. À cause de la variabilité dans le programme d'inventaire, certaines placettes ont été inventoriées à deux reprises au cours de la même décennie, particulièrement durant les années 1970; ceci fut pris en compte pour l'analyse statistique (plus bas). La date moyenne des relevés pour chaque décennie fut respectivement : 1975,0 ( $\mathrm{n}=9948)$, $1984,7(n=5347)$ et $1995,8(n=7240)$.

Dans chaque placette, on détermine l'âge de neuf arbres en comptant les anneaux de croissance à une hauteur de $1 \mathrm{~m} \mathrm{du}$ sol à l'aide d'une sonde de Pressler; cinq sont choisis au hasard et quatre pour leur représentativité des tiges dominantes et codominantes (Anonyme 2001a). Lorsqu'une parcelle ne contient pas suffisamment d'arbres représentatifs, on en sélectionne à l'extérieur de celle-ci. Lorsqu'un peuplement est bi-étagé, on détermine l'âge d'arbres représentatifs des deux étages. On compte les anneaux de croissance immédiatement sur le terrain et une vérification suit au laboratoire. On assigne ensuite une classe d'âge à chaque peuplement, normalement par tranche de 20 ans. On ne détermine toutefois pas l'âge des feuillus autres que les peupliers et le bouleau à papier (Betula papyrifera) de sorte qu'on estime l'âge des peuplements feuillus à partir de l'âge des conifères présents ou d'autres indices; tous les peuplements se voient assigner une classe d'âge. Ainsi l'âge des peuplements ne réfere pas au temps écoulé depuis la dernière perturbation majeure, mais à l'âge de la cohorte d'arbres dominants. Nous avons regroupé les classes d'âge en trois pour notre analyse : peuplements jeunes $(<60$ ans), matures (60-100 ans) et âgés ( $>101$ ans). Les peuplements classés «jeunes irréguliers» ont été assignés à la classe $<60$ ans alors que les peuplements de classe «vieux irréguliers» le furent à la classe $>100$ ans (Crête et al. 2004a). Quand un peuplement comptait deux étages, nous avons utilisé l'âge de l'étage le plus vieux (Crête et al. 2004a).

Lors du relevé, on dénombre, par espèce, tous les arbres vivants contenus à l'intérieur de la placette principale et on mesure leur DHP à une précision de $1 \mathrm{~mm}$. On dénombre et on mesure également le DHP des arbres morts récemment (chicots), soit les coniferes morts avec un fin bout $<5 \mathrm{~cm}$ et les feuillus morts portant encore la plupart de leurs branches principales. De la même manière, on dénombre, par espèce, les gaules présentes dans les placettes de $40 \mathrm{~m}^{2}$. On note enfin la présence de signes de perturbation qui sont à l'origine du peuplement ou qui l'ont partiellement affecté, notamment la 
coupe (souches visibles, chemin de débardage, etc.), le feu (troncs, souches ou débris ligneux calcinés) ou les épidémies d'insectes (défoliation, arbres moribonds ou morts avec leurs branches, troncs au sol sans autres causes probables).

Nous avons utilisé les relevés aériens effectués par la Direction de la conservation des forêts afin de calculer, par décennie et sous-domaine bioclimatique, un indice d'infestation des forêts par la tordeuse des bourgeons de l'épinette (Choristoneura fumiferana), un insecte ayant tué un grand nombre d'arbres durant la période d'étude. La proportion annuelle moyenne par décennie de la superficie de chaque sous-domaine affectée par une infestation grave a servi d'indice d'activité de la tordeuse.

\section{Analyse statistique}

Pour les variables continues (nombre d'espèces d'arbres et de gaules par station, proportion d'arbres et de gaules feuillues), nous avons utilisé une analyse de variance à mesures répétées pour déterminer si les variables d'intérêt différaient en fonction des facteurs principaux (sous-domaines bioclimatiques, âge du peuplement (jeune, mature, suranné) et décennie), ainsi que de leurs interactions. Comme il était difficile de normaliser les résidus par des transformations, nous avons pratiqué les ANOVA sur les valeurs transformées en rang. Comme il y avait possibilité de dépendance entre les deux placettes d'une même virée (allocation aléatoire par grappe de deux), nous avons pratiqué les ANOVA sur la moyenne des deux placettes de chaque grappe. Pour les variables catégoriques (espèces d'arbres ou de gaules), nous avons utilisé des modèles log-linéaires incluant les mêmes variables explicatives et leurs interactions que pour l'ANOVA; nous avons repris l'analyse avec une seule placette par grappe de deux pour s'assurer que la dépendance ne biaisait pas l'analyse. Nous avons pratiqué quelques analyses de régression linéaire simple ou multiple afin d'évaluer la force du lien existant entre la variable dépendante et les variables explicatives; nous nous sommes assurés que les résidus possédaient une distribution normale à l'aide du test de Shapiro-Wilk et qu'ils ne montraient pas de patron grâce à un examen visuel du graphique. Comme la composition des forêts varie progressivement en passant d'un domaine bioclimatique à l'autre, nous avons pratiqué une analyse statistique séparée pour chaque domaine bioclimatique en contrastant la partie est et ouest de chacun, dans les cinq cas où cette subdivision existait (Fig. 1). Nous présentons tous les résultats sous forme d'histogrammes dont la disposition respecte la position géographique de chaque sous-domaine bioclimatique pour faciliter l'interprétation des résultats; bien que la taille des effectifs ait différé légèrement d'une variable à l'autre à cause de données manquantes, nous ne présentons les effectifs que sur la première figure pour alléger le contenu présentation. Le progiciel SAS (SAS Institute 1999) a servi pour toutes les analyses statistiques.

\section{Résultats \\ Les forêts âgées}

Dans les forêts québécoises de la fin du $20^{\mathrm{e}}$ siècle, les peuplements âgés ne s'avéraient communs que dans la pessière à mousses alors que, déjà dans les années 1970, ils représentaient moins de $10 \%$ des surfaces forestières dans plusieurs autres sous-domaines, notamment ceux de la sapinière à bouleau blanc. Les forêts étaient plus jeunes dans la partie est des sapinières et des érablières, alors que l'inverse s'observait dans la pessière à mousses. Au cours des trois dernières décennies, la structure d'âge des peuplements forestiers, regroupés en trois classes - jeune, mature, âgée a varié dans tous les domaines bioclimatiques (Fig. 2). La raréfaction des vieux peuplements se produisit uniquement dans la sapinière à bouleau jaune, notamment dans l'ouest, ainsi que dans l'érablière à bouleau jaune de l'ouest. La proportion de peuplements âgés demeura faible mais relativement stable dans la sapinière à bouleau blanc et l'érablière à tilleul; dans ce dernier domaine, les peuplements matures (60-100 ans) gagnèrent de l'importance durant la période d'étude. Dans la pessière, la proportion de vieux peuplements tendit à s'accroître.

\section{Le bois mort}

Il y avait, en moyenne, entre $\approx 20$ et $\approx 80$ chicots récents/ha $($ DHP $>9 \mathrm{~cm})$ dans l'ensemble des forêts du Québec au cours des trois dernières décennies (Fig. 3). Le nombre moyen de gros chicots $(\mathrm{DHP}>20 \mathrm{~cm})$ était relié directement au nombre moyen total de chicots pour l'ensemble des sousdomaines bioclimatiques $\left(\mathrm{R}^{2}\right.$ ajusté $\left.=0,82 ; \mathrm{df}=32 ; \mathrm{P}<0,001\right)$. L'évolution de la densité de chicots récents au cours des décennies a montré des tendances différentes dans les forêts feuillues du sud (domaine des érablières) et les forêts de coniferes (sapinières et pessière), et est demeurée, de façon générale, stable dans les forêts feuillues. On a quand même dénombré plus de chicots au cours de la décennie 1980 dans les érablières à tilleul et à bouleau jaune de l'est, augmentation qui n'est pas survenue dans l'ouest. Dans l'érablière et la sapinière à bouleau jaune de l'ouest, il y eut une tendance à une raréfaction des chicots au cours de l'étude. Durant les années 1970, on a compté plus de chicots dans les sapinières de l'ouest, alors que l'augmentation des années 1980 a été beaucoup plus marquée dans les sapinières de l'est. Dans les pessières, la densité de chicots a augmenté progressivement au fil des décennies dans l'est, alors qu'on a observé un creux au cours des années 1980 dans l'ouest. On a observé une relation positive entre la densité moyenne de chicots récents et la proportion de placettes où les techniciens notèrent des signes d'une épidémie d'insectes au cours de la même décennie $\left(\mathrm{R}_{\text {ajusté }}=0,17 ; \mathrm{df}=32 ; \mathrm{P}=0,010\right)$. La meilleure relation liant la densité de chicots et l'épidémie de tordeuse provint, cependant, des compilations des relevés aériens, en prenant en compte le pourcentage du sous-domaine affecté sévèrement au cours de la même décennie et de la décennie antérieure $\left(\mathrm{R}_{\text {ajusté }}^{2}=0,45 ; \mathrm{df}=21 ; \mathrm{P}=0,001\right)$.

\section{La composition et la diversité des forêts}

Les arbres feuillus perdent de l'importance en progressant vers le nord, passant de $60-70 \%$ dans les forêts du sud du Québec à 10-20\% dans la pessière à mousses (Fig. 4). Leur proportion n'a pas varié de façon significative en fonction de la décennie ou de son interaction avec les autres facteurs principaux, sauf dans l'érablière à caryer et l'érablière à bouleau jaune. Cependant, la proportion de feuillus varia partout en fonction des trois classes d'âge. Dans les sapinières et la pessière, il existait une tendance pour que la proportion de feuillus diminue avec l'âge. En général, il y avait plus de feuillus dans la partie ouest des sous-domaines, sauf pour la 


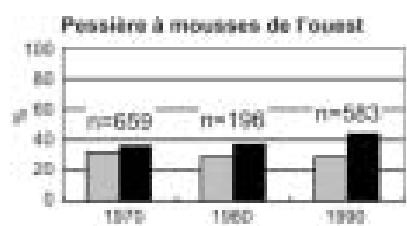

$0=0,006$

$S D<0,001$



$D<0,00\}$

$S D=0005$

$D \times .9 D=0,001$

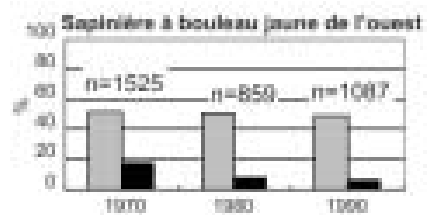

$\mathcal{D}<0,001$

$S D<0,001$

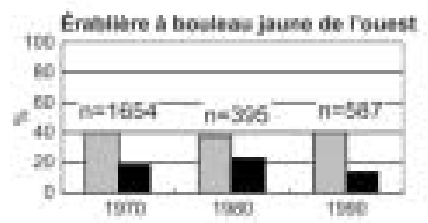

$0<0,002$

$S W<0,001$

$D \times S D=0,001$

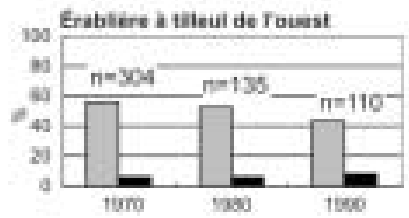

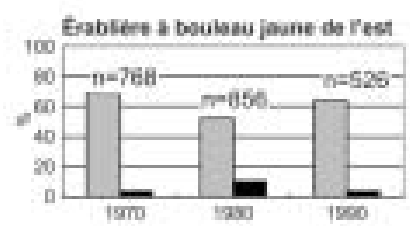


$D=0,006$

$9 D<0,00 t$

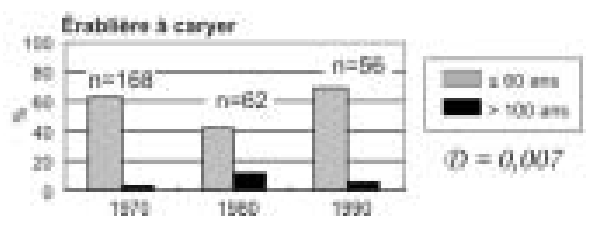

Fig. 2. Proportion des peuplements forestiers classés jeunes ( $<60$ ans) et âgés ( $>100$ ans) dans les six domaines bioclimatiques du sud du Québec au cours des trois dernières décennies. L'analyse statistique pour chaque domaine indique la probabilité que la structure d'âge des peuplements ait varié en fonction de la décennie (D), du sous-domaine (SD) ou de leur interaction (D × SD).

pessière où la tendance s'inversait. Dans les érablières, les feuillus tendirent à prendre de l'importance durant la décennie 1980. La proportion de tiges feuillues a varié plus clairement en fonction des décennies chez les gaules que chez les arbres dans les sapinières et la pessière (Fig. 5). On observa une nette augmentation de l'importance des gaules feuillues dans les sapinières et la pessière à mousses de l'ouest, plus particulièrement pour les classes d'âge $<60$ ans et $60-100$ ans. La proportion de gaules et d'arbres feuillus a varié en parallèle au fil des décennies, dans l'ensemble des sous-domaines (Fig. 4 et $\left.5: \mathrm{R}_{\text {ajusté }}^{2}=0,76 ; \mathrm{df}=95 ; \mathrm{P}<0,001\right)$.
La dominance d'une ou deux espèces d'arbres dans les forêts du Québec s'est accentuée en progressant de l'érablière à caryer vers la pessière à mousses (Fig. 6). Le sapin baumier représenta l'espèce la plus ubiquiste, alors que l'érable rouge (Acer rubrum) s'avéra particulièrement abondant dans le sud. Dans les forêts du nord, le bouleau à papier fut deux à quatre fois plus abondant que le peuplier faux-tremble (Populus tremuloides), alors que dans les érablières, l'érable à sucre (Acer saccharum) était plus commun dans l'ouest que dans l'est. La composition des peuplements forestiers a varié en fonction des décennies dans tous les sous-domaines 


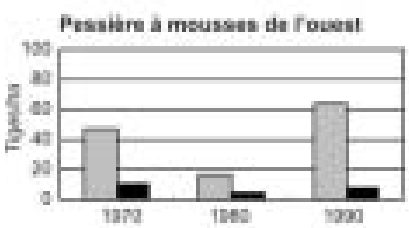

$D=0,010$

$S D=0,0 \mathrm{t}$

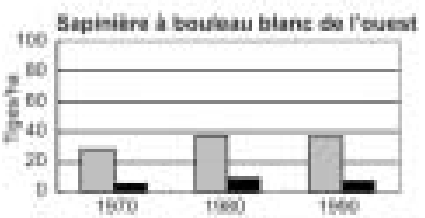

$0=0,001$

$S D=0,008$

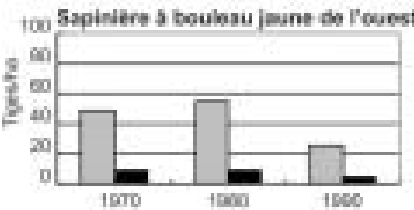

$D=0,009$

$S D=0,000$

$D \times S D=0,007$

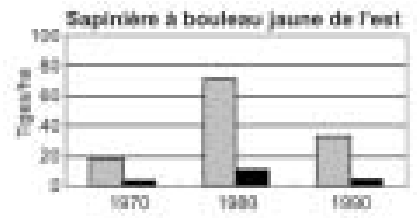

Erabidie a beulew jaune de fouss

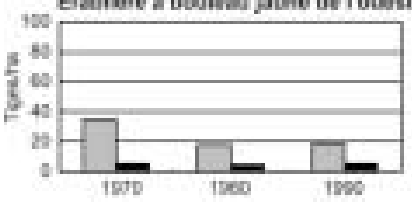

$9 D=0007$

$D \times S D<0,001$


$D \times S D=0.012$

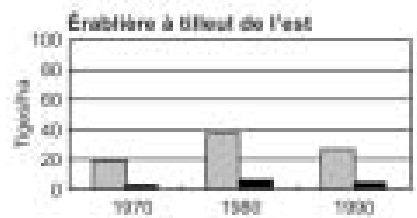

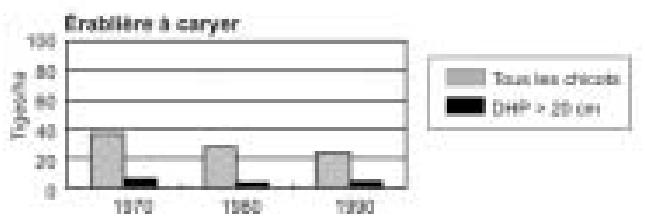

Fig. 3. Densité moyenne de chicots récents de toute dimension et de gros chicots (DHP > $20 \mathrm{~cm}$ ) observée au cours des trois dernières décennies en fonction des six domaines bioclimatiques couvrant le sud du Québec. L'analyse statistique pour chaque domaine indique la probabilité que la densité de chicots de toute taille ait variée en fonction de la décennie (D), du sous-domaine (SD) ou de leur interaction (D $\times$ SD)

$(\mathrm{P}<0,001)$. Le sapin baumier a clairement perdu de l'importance dans les sapinières et la pessière à mousses de l'ouest, au profit du bouleau à papier, de l'épinette noire et des autres espèces. L’importance relative des espèces de gaules a également changé en fonction des décennies dans tous les sous-domaines $(\mathrm{P}<0,001$; Fig. 7$)$. On a observé une perte assez généralisée de l'importance relative des gaules de sapin dont la place fut comblée par des essences secondaires, notamment l'érable à épis (Acer spicatum), l'aulne rugueux (Alnus rugosa), le sorbier d'Amérique (Sorbus americana). L'érable à sucre a également eu tendance à se raréfier au cours de la dernière décennie. Chez les gaules, on a observé une relation négative très claire entre le pourcentage de feuillus dans les peuplements et celui du sapin baumier $\left(\mathrm{R}^{2}{ }_{\text {ajusté }}=\right.$ $0,48 ; \mathrm{df}=32 ; \mathrm{P}<0,001)$, une relation beaucoup moins nette chez les arbres $\left(\mathrm{R}_{\text {ajusté }}^{2}=0,06 ; \mathrm{df}=32 ; \mathrm{P}=0,094\right)$.

Le nombre moyen d'espèces d'arbres et de gaules n'a jamais dépassé cinq par placette (Fig. 8). La diversité des arbres la plus grande s'observa dans l'érablière à bouleau jaune. Cette variable demeura relativement constante au fil des décennies dans la sapinière à bouleau jaune et les autres domaines situés plus au sud, avec une tendance pour une diversité plus grande dans la partie ouest des sous-domaines. Dans la sapinière à bouleau blanc et la pessière, la diversité en 


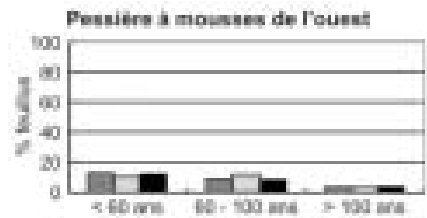

\section{$\lambda=0,001$}

$s w=0,009$

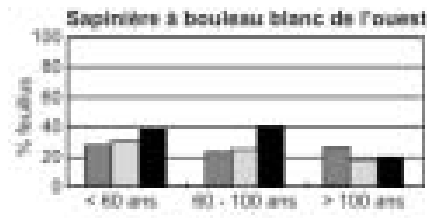

$A=0,039$

$S D=0,0216$
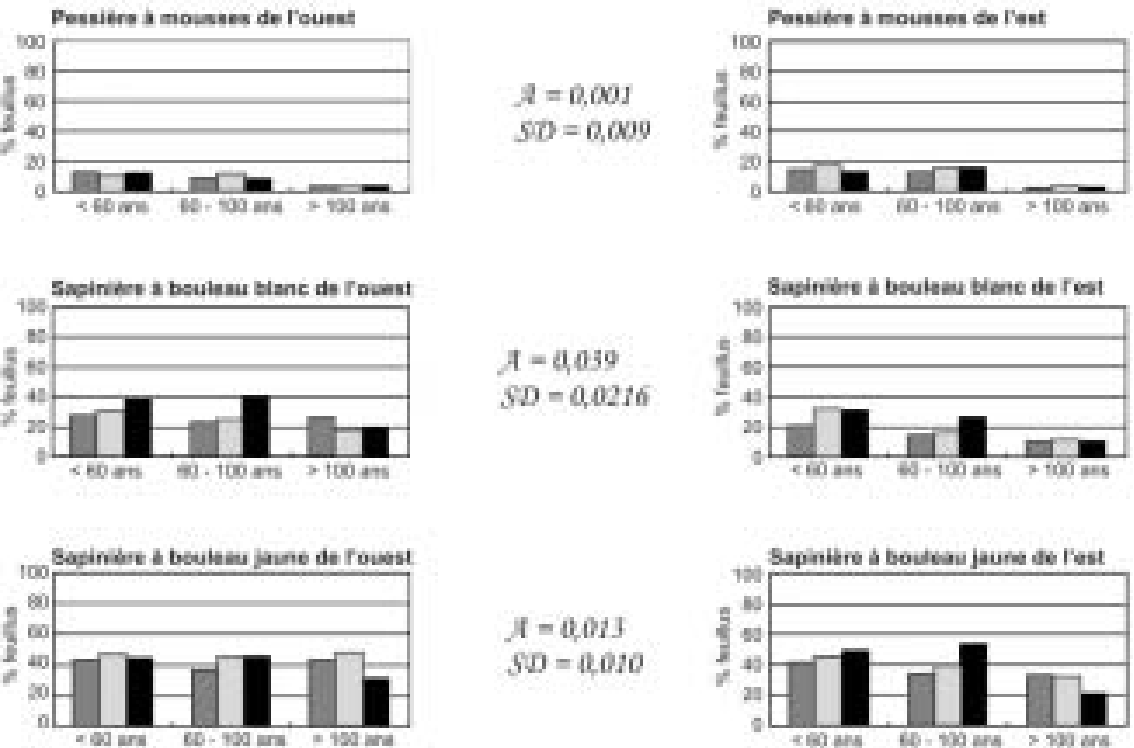

$t=0,013$

$S D=Q, Q H O$
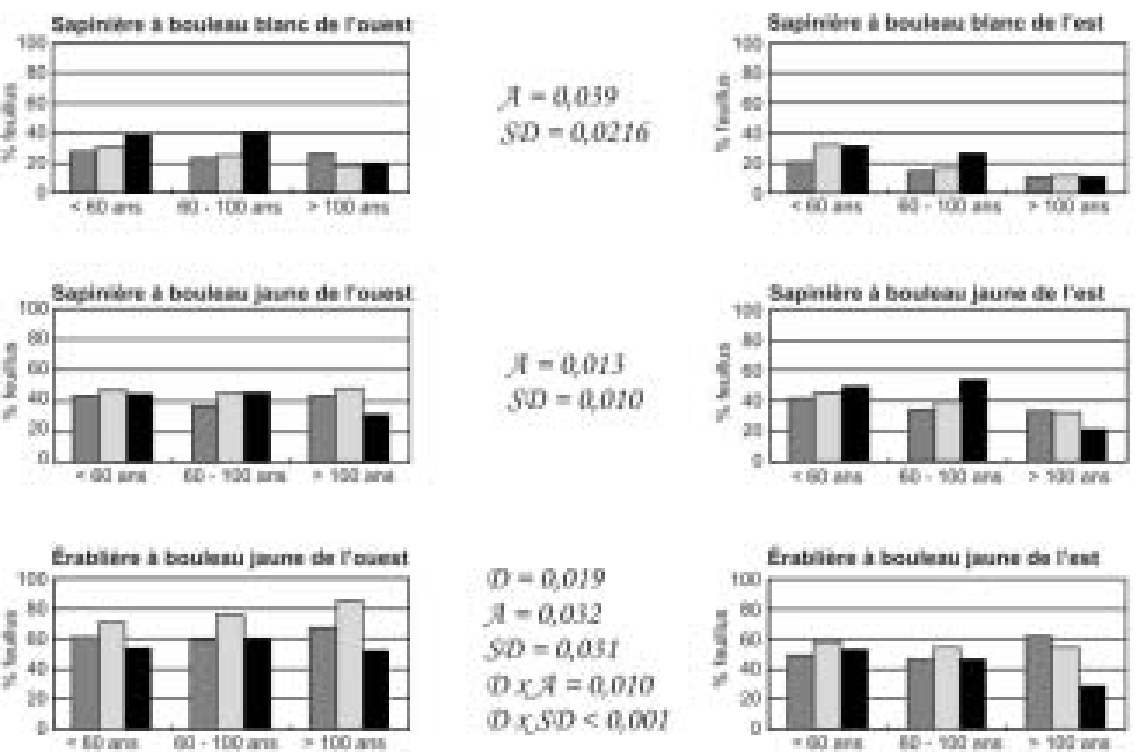

$0=0.019$

$A=0,032$

$S D=0,031$

D. $x, A=0,010$

$D \times S D<0,001$
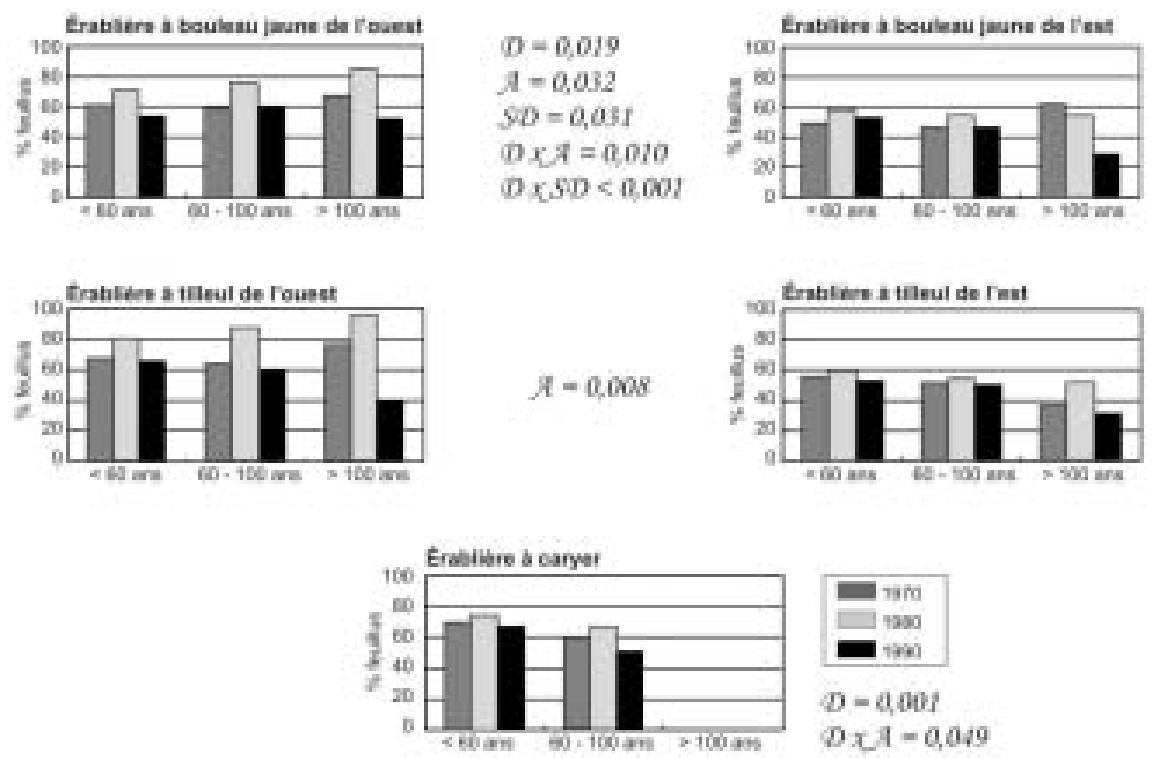

Fig. 4. Pourcentage de feuillus observé chez les arbres (DHP> $9 \mathrm{~cm}$ ) au cours des trois dernières décennies en fonction de trois classes d'âge et des six domaines bioclimatiques couvrant le sud du Québec. L'analyse statistique pour chaque domaine indique la probabilité que la proportion de feuillus ait varié en fonction de la classe d'âge (A), de la décennie (D), du sous-domaine (SD) ou des interactions de second degré. L'effectif était trop petit pour calculer un pourcentage dans les peuplements âgés de l'érabière à caryer.

arbres tendit à diminuer au cours des années 1980 dans la partie ouest des domaines. La diversité en gaules s'avéra légèrement plus grande dans les sapinières que dans les autres domaines bioclimatiques, particulièrement durant les années 1990 (Fig. 8). La diversité en gaules changea partout en fonction des décennies, sauf dans les deux domaines les plus au sud où elle demeura stable et faible. La diversité en gaules eut souvent tendance à augmenter au cours des années 1990, mais elle ne varia pas de façon symétrique entre les parties ouest et est des domaines bioclimatiques. On a observé une relation très nette et positive entre la densité des gaules et le nombre d'espèces recensées par station pour l'ensemble des domaines bioclimatique au cours des trois décennies $\left(\mathrm{R}_{\text {ajusté }}^{2}\right.$ $=0,80 ; \mathrm{df}=32 ; \mathrm{P}<0,001)$.

\section{Discussion}

Nous avions prédit que l'intensification de l'exploitation forestière au cours des trois dernières décennies au Québec conduirait à une raréfaction des forêts âgées et du bois mort, ainsi qu'à une réduction de la diversité des arbres et des arbustes, en présumant que l'aménagement forestier représentait le facteur de perturbation dominant le paysage. 


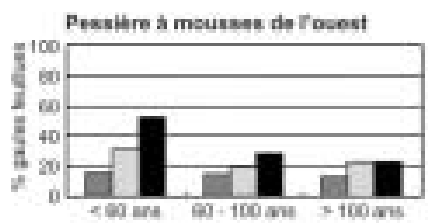

$D=0,016$

$\lambda=0,002$

$D, S D=0,04\}$

$A \times 5 D=0,050$
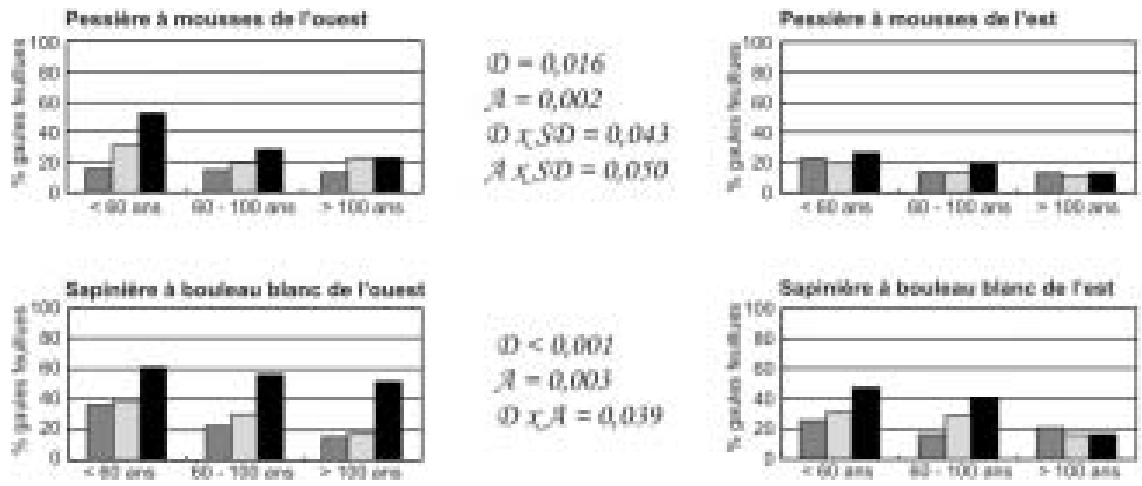

$0<0,001$

$\lambda=0,003$

$0 \times \mathcal{A}=0,039$
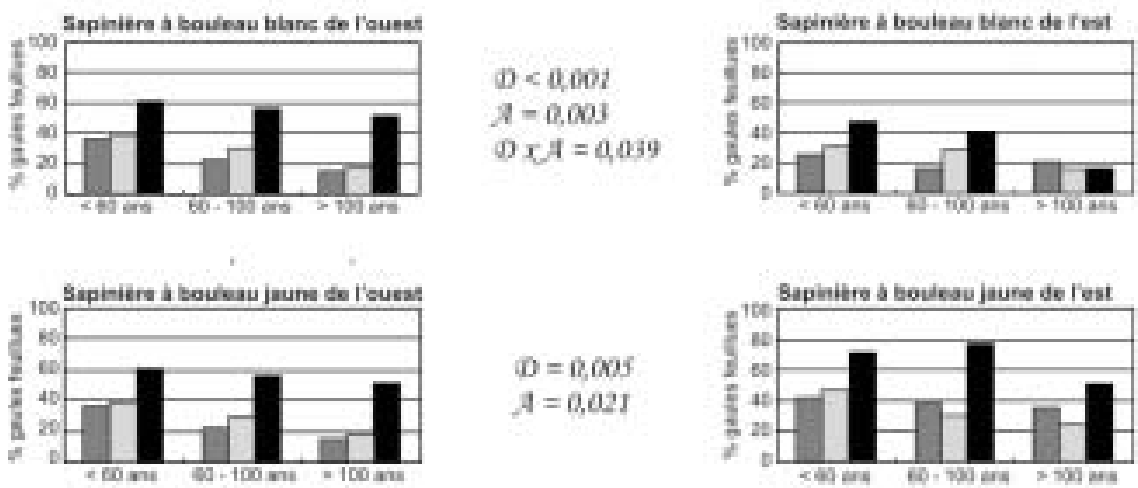

$\omega=0,005$

$A=0,021$
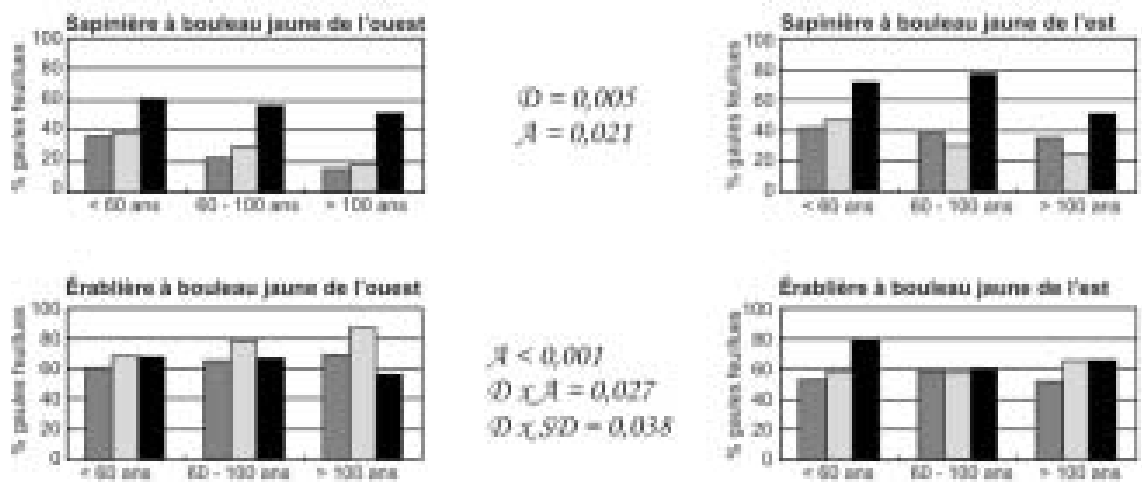

$A<0,00\rangle$

D) $\mathrm{A}=0,027$

$D \times .5 D=0,038$
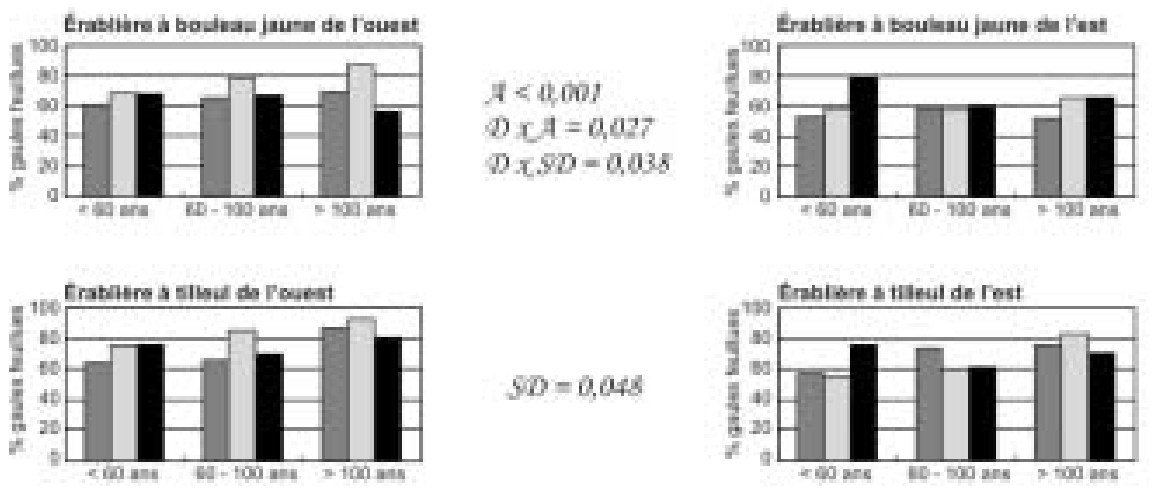

$S D=0,048$
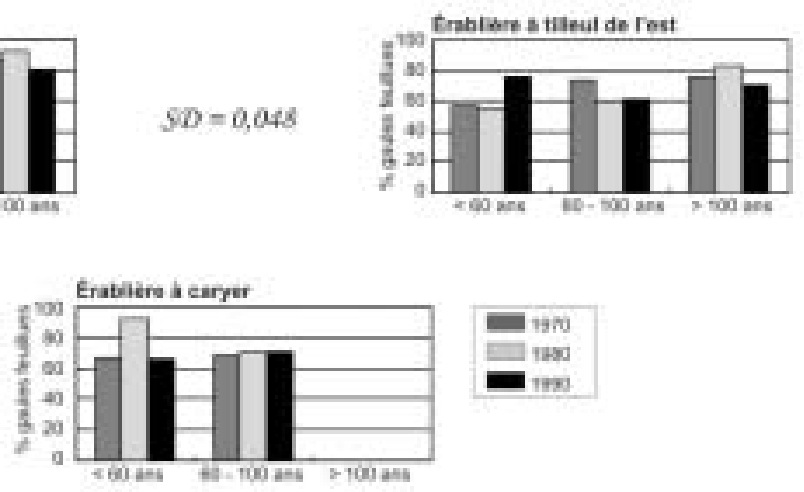

Fig. 5. Pourcentage de feuillus observé chez les gaules $(1 \mathrm{~cm}<\mathrm{DHP} \leq 9 \mathrm{~cm})$ au cours des trois dernières décennies en fonction de trois classes d'âge et des six domaines bioclimatiques couvrant le sud du Québec. L'analyse statistique pour chaque domaine indique la probabilité que la proportion de feuillus ait varié en fonction de la classe d'âge (A), de la décennie (D), de la classe d'âge (A), du sous-domaine (SD) ou des interactions de second degré. L'effectif était trop petit pour calculer un pourcentage dans l'érabière à caryer.

Nous avons utilisé un puissant réseau de 7000 placettes permanentes pour tester nos prédictions et force est de conclure que l'évolution des forêts s'est avérée plus complexe que nous l'imaginions. Il faut examiner chaque variable attentivement pour cerner ce qui est survenu.

\section{Les forêts âgées}

Nous avons utilisé l'âge des arbres composant la cohorte dominante pour établir l'âge des peuplements, ce qui ne correspond pas nécessairement au temps écoulé depuis la dernière perturbation majeure. Ainsi, la proportion de peuplements âgés que nous présentons représente une sous-estimation en termes de date d'établissement des peuplements.

Durant la période d'étude, les forêts âgées étaient concentrées principalement dans le domaine de la pessière à mousses. Ceci s'explique par l'arrivée récente de l'exploitation forestière dans ce domaine éloigné des bassins de population et des usines de transformation du bois. Ainsi, le régime de perturbations naturelles, principalement le feu, y dominait la dynamique forestière jusqu'à tout récemment (Bergeron et al. 2001). Nos résultats concernant la proportion de peuple- 

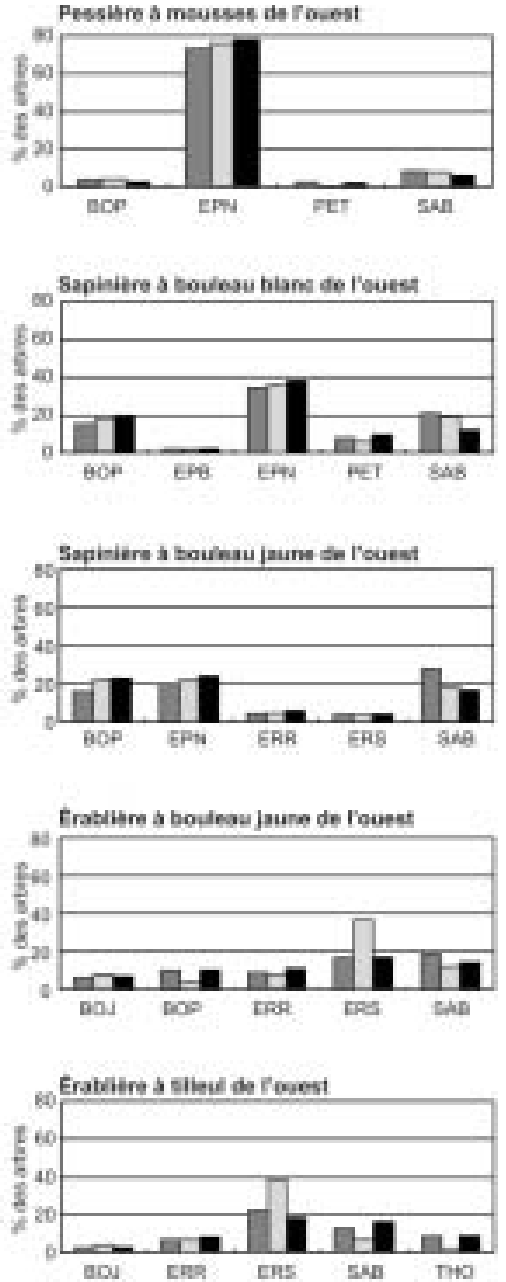
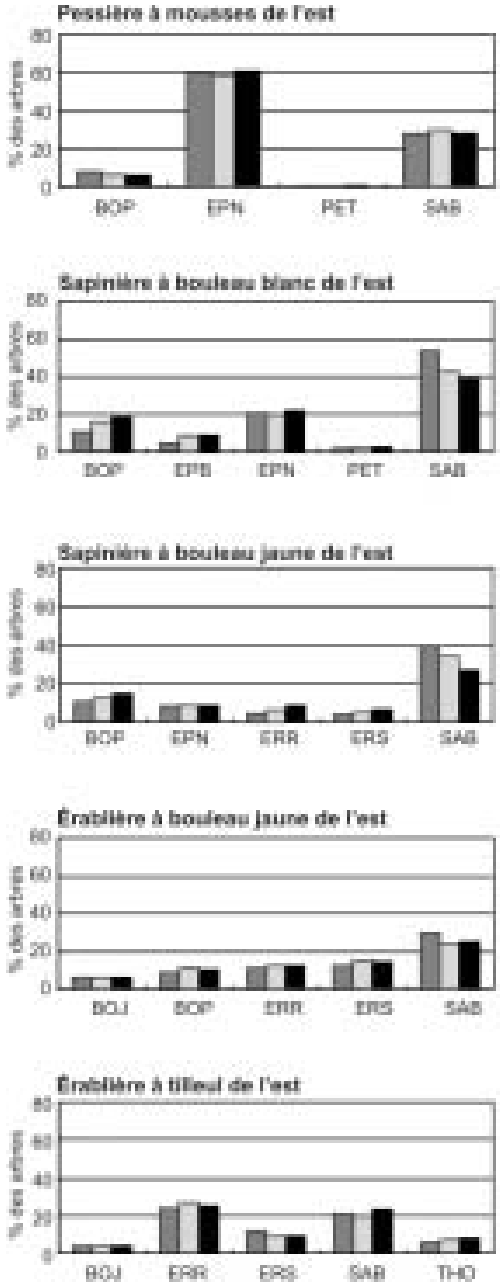

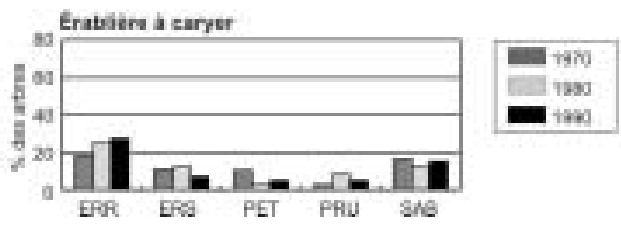

Fig. 6. Importance relative des quatre ou cinq espèces d'arbres (DHP $>9 \mathrm{~cm}$ ) les plus importantes observées au cours des trois dernières décennies en fonction des six domaines bioclimatiques couvrant le sud du Québec. BOJ = Betula alleghaniensis; BOP = Betula papyrifera; EPB = Picea glauca; EPN = Picea mariana; ERR = Acer rubrum; ERS = Acer saccharum; PET = Populus tremuloides; PRU = Tsuga canadensis; $\mathrm{SAB}=$ Abies balsamea; THO = Thuja occidentalis

ments âgés dans ce domaine doivent toutefois être nuancés parce que près de $20 \%$ des placettes permanentes se situaient à l'extérieur des forêts aménagées. Dans la portion aménagée de la pessière à mousses, l'exploitation s'est accélérée durant la période d'étude. Ainsi, les peuplements âgés seront concentrés de plus en plus dans la portion inexploitée de la pessière à mousses si la tendance observée se maintient. L'augmentation de l'importance relative des peuplements âgés au cours de la période d'étude dans la pessière reflète possiblement l'allongement du cycle des feux qu'on a observé dans la forêt boréale québécoise depuis le milieu du $19^{\mathrm{e}}$ siècle
(Bergeron et al. 2001). Ainsi, plusieurs peuplements apparus entre 1870 et 1890 pourraient être passés dans la classe d'âge de 120 ans au cours de la période d'étude. Par ailleurs, la plus grande proportion de peuplements âgés dans l'est que dans l'ouest de la pessière à mousses illustre que le cycle des feux a été plus long dans la partie orientale de ce domaine au cours des derniers siècles (Bergeron et al. 2001).

Dans les années 1970, les peuplements âgés s'avéraient également assez communs dans l'érablière et la sapinière à bouleau jaune de l'ouest. Toutefois, dans les domaines où les espèces feuillues sont répandues, il peut exister une sous- 

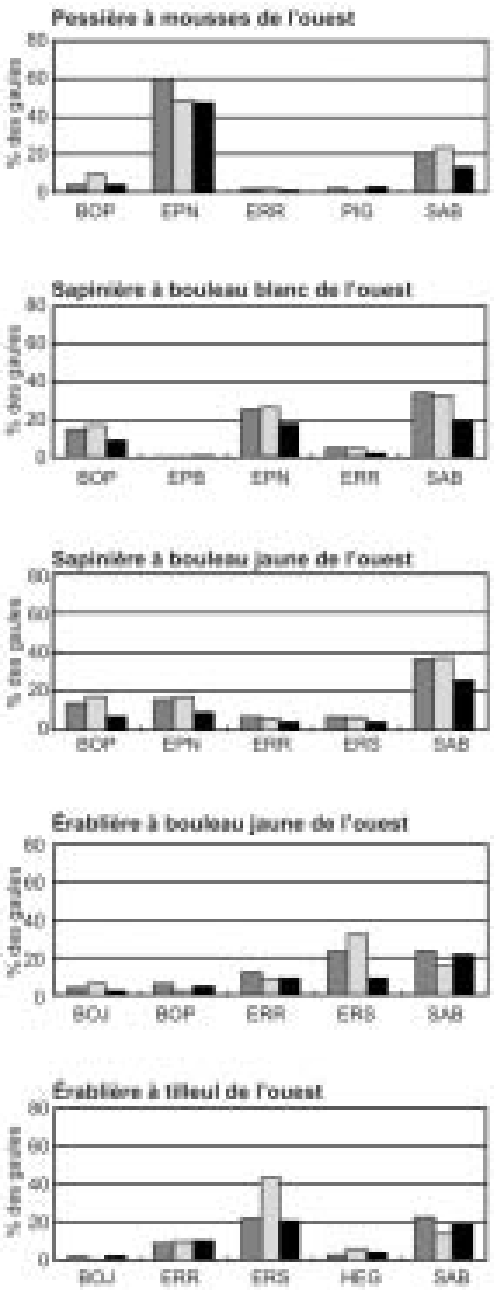
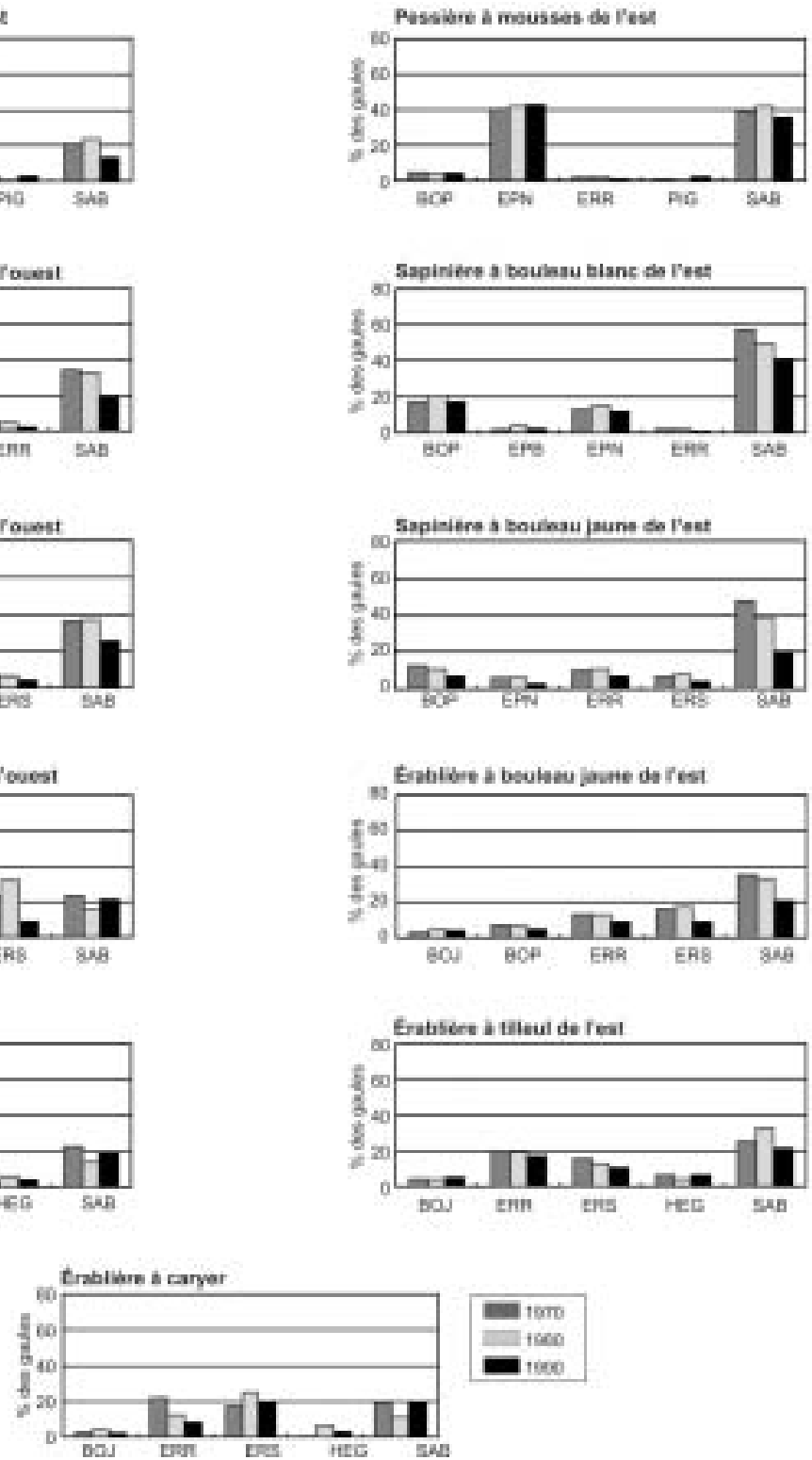

Fig. 7. Importance relative des cinq espèces de gaules (DHP $<9 \mathrm{~cm}$ ) les plus importantes observées au cours des trois dernières décennies en fonction des six domaines bioclimatiques couvrant le sud du Québec. BOJ = Betula alleghaniensis; BOP = Betula papyrifera; EPB = Picea glauca; EPN = Picea mariana; ERR = Acer rubrum; ERS = Acer saccharum; HEG = Fagus grandifolia; PIG = Pinus banksiana; SAB = Abies balsamea

estimation de l'importance des peuplements âgés si la longévité des feuillus surpassait celle des coniferes parce que ce sont surtout les coniferes qui ont servi à établir l'âge des peuplements. Les peuplements âgés ont nettement régressé, durant la période d'étude, dans la sapinière et l'érablière à bouleau jaune de l'ouest, vraisemblablement à cause de l'intensification de l'exploitation forestière couplée à l'épidémie de tordeuse des bourgeons de l'épinette. Crête et al. (2004a) ont d'ailleurs trouvé moins de 3\% de forêts âgées dans un territoire de plus de $1000 \mathrm{~km}^{2}$ de ces domaines au début des années 2000 et ont noté que l'exploitation forestière s'était intensifiée au cours des 10 dernières années.

Ailleurs, il y avait moins de $10 \%$ de peuplements âgés pendant toute la période d'étude. Toutefois, les sapinières âgées pourraient s'avérer rares même sous un régime de perturbations naturelles puisque la tordeuse des bourgeons de l'épinette y a fait éruption à intervalle d'environ 40 ans depuis au moins 450 ans (Boulanger et Arsenault 2004). Enfin, l'empreinte d'une occupation humaine du territoire plus dense dans l'est que dans l'ouest de l'érablière et de la 


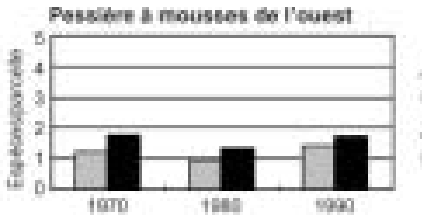

Anings

$0=\pi$.

$S D=0,00 t$

$0 \times 5 z=0,009$

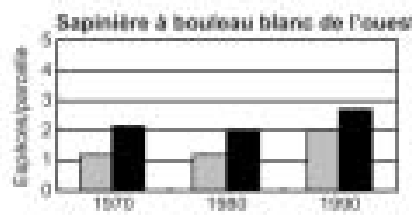

Anfws

$D=0,0<2$

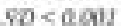

D. $.50=4001$

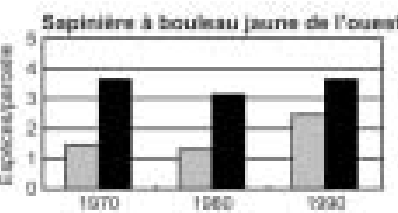

\section{Anores}

$D=\pi-s$.

SD $=0.028$

$D \times S D=n$.S

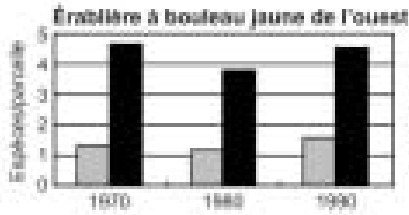

\section{Antries}

$D=k x$

SD = Qove

$0 \times 500=0,002$

\section{Gamiss}

$<0,009$

$=2,003$

tis

Crates

$<0,001$

- ander

$-0,00 r$

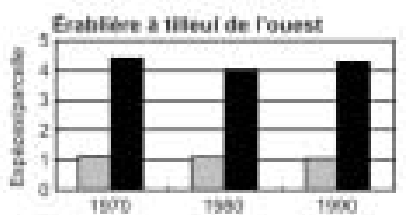

Anorys

$D=n$. S.

(5) $=0$ ant

D.520 4 a.s

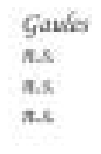

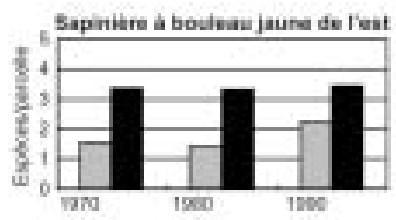

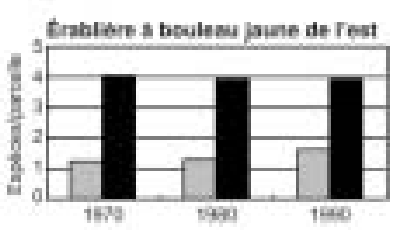

Pessibre a mousess de Test

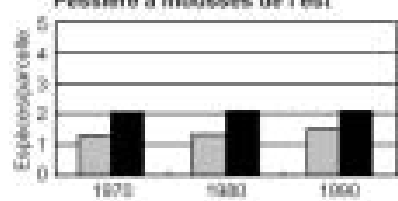

Baginere a boulanu biane de fest
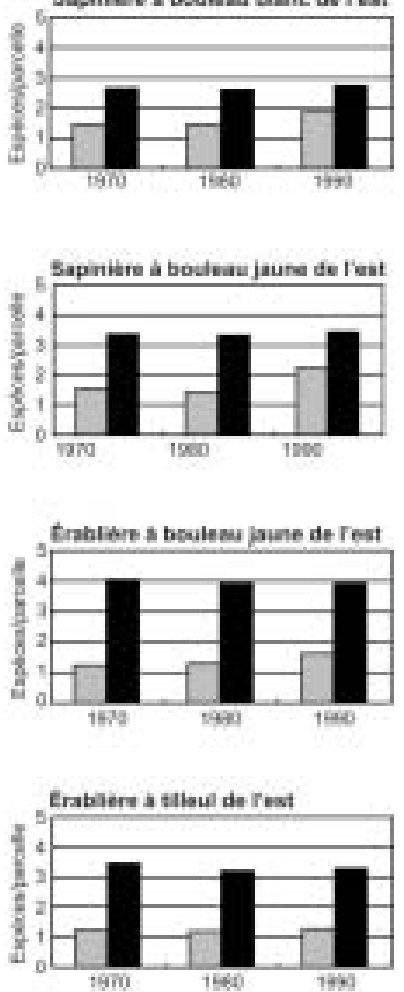

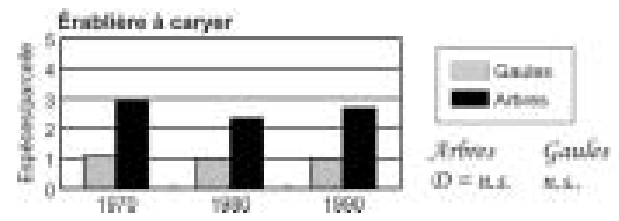

Fig. 8. Nombre moyen d'espèces d'arbre $\left(400 \mathrm{~m}^{2}\right.$ ) et de gaules $\left(40 \mathrm{~m}^{2}\right.$ ) observé par placette au cours des trois dernières décennies en fonction des six domaines bioclimatiques couvrant le sud du Québec. L'analyse statistique pour chaque domaine indique la probabilité que la diversité en espèces ait variée en fonction de la décennie (D), du sous-domaine (SD) ou de leur interaction ( $\times$ × SD). N.S. = non significatif.

sapinière à bouleau jaune s'est reflétée par la plus grande rareté de peuplements âgés dans la partie orientale de ces domaines.

Une analyse récente de l'évolution des forêts québécoises est arrivée à une constatation qui differe quelque peu de la nôtre en ce qui concerne les forêts âgées (Anonyme 2004). Cette analyse, basée sur la photointerprétation et la cartographie, a mis en évidence une raréfaction des forêts âgées dans la pessière à mousses et à une augmentation de leur importance dans la sapinière et l'érablière à bouleau jaune de l'ouest. L'écart entre les deux analyses tient vraisemblablement à un regroupement différent des classes d'âge (peuplements âgés (> 100 ans) contre peuplements mûrs et surannés), à la base de données (placettes permanentes contre cartographie SIFORT) et à l'aire d'étude (aire échantillonnée par les placettes permanentes contre forêts publiques sous aménagement). Il est difficile de déterminer quelle base de données fournit la meilleure image de l'évolution de l'abondance des vieilles forêts au Québec, mais on sait que la photointerprétation conduit à des erreurs qui 
n'existent pas pour des relevés de terrain (Potvin et al. 1999, Dussaut et al. 2001, Lesage et al. 2002). De plus, l'approche cartographique ne prend pas en compte que les peuplements inférieurs 4 ou 8 ha, ce qui biaise à la baisse l'importance de petites unités (p. ex. des séparateurs de coupe ou des bandes riveraines) dans un paysage de forêts aménagées.

Déjà dans les années 1970, les habitats forestiers favorisaient les espèces animales recherchant les jeunes forêts. La raréfaction des vieilles forêts s'est donc vraisemblablement réalisée avant la mise sur pied du réseau de placettes permanentes. À ce jour, nous connaissons peu d'espèces de la faune forestière québécoise que la raréfaction en soi des peuplements âgés mettrait en danger. L'aire de répartition du caribou forestier (Rangifer tarandus) a reculé partout au Canada face à la progression de la coupe (Schaefer 2003), sauf à Terre-Neuve où l'espèce a atteint de fortes densités en présence d'exploitation forestière (p. ex. Mahoney et Virgl 2003). Le caribou forestier a également régressé au Québec au cours du dernier siècle (Courtois et al. 2003). Récemment, les caribous forestiers recherchaient les massifs de peuplements âgés dans la pessière à mousses de l'est, mais se sont maintenus dans des paysages partiellement rajeunis par la coupe forestière (Courtois 2003). Il est difficile de départager l'effet direct de la transformation de l'habitat du caribou forestier causée par la coupe des effets indirects. On sait que le caribou est très sensible aux activités et aux installations humaines (Nelleman et Cameron 1996; Bradshaw et al. 1997; Maier et al. 1998; Nelleman et al. 2000, 2001; Dyer et al. 2001, 2002; Mahoney et Schaefer 2002; Reimers et al. 2003); or, l'exploitation forestière accroit le dérangement parce qu'elle augmente l'accessibilité, et conséquemment la villégiature, la circulation, la chasse, etc. (Mahoney et Virgl 2003; Courtois et al. 2004). L'exploitation forestière pourrait aussi favoriser l'accroissement de la densité d'orignaux (Alces alces) par une production accrue de brout, qui amènerait dans sa suite davantage de loups gris (Canis lupus), un prédateur du caribou (Bergerud et Elliot 1986, Seip 1992). Toutefois, aucune donnée ne supporte encore cette dernière hypothèse pour le Québec. On a également présenté la martre d'Amérique (Martes americana) comme une espèce typique des forêts de coniferes mûres et surannées (Thompson 1991). Toutefois, Potvin et al. (2000) ont observé que cette espèce pouvait se satisfaire d'un habitat fragmenté par la coupe, en autant que les peuplements coupés depuis moins de 30 ans n'excèdent pas 30\%. En Colombie Britannique, Poole et al. (2004) ont étudié une population de martre vigoureuse occupant des friches agricoles vieilles de 30 à 40 ans et régénérées en feuillus. Par ailleurs, Drapeau et al. (2003) ont étudié les communautés d'oiseaux occupant des peuplements mûrs et surannés de la pessière à mousses de l'ouest; les communautés différaient en fonction de l'âge des peuplements, notamment à cause d'une structure différente, mais aucune espèce ne se restreignait aux forêts surannées. Plusieurs espèces animales, notamment les pics (Imbeau et al. 2001), dépendent du bois mort qui abonde généralement dans les forêts surannées (Desponts et al. 2004). Il est cependant encore difficile de départager le rôle respectif joué par les peuplements surannés et le bois mort en soi pour leur conservation. Les espèces les plus à risque face à la raréfaction des peuplements âgés se retrouvent vraisemblablement chez les invertébrés, à cause d'une capacité de dispersion souvent limitée; la découverte des espèces potentiellement à risque parmi plus de 25000 espèces méconnues s'avère toutefois un tâche ardue. Il faudrait notamment porter une attention particulière aux pessières à mousses surannées de l'ouest qui subissent de la paludification en vieillissant (Harper et al. 2005), ce qui en fait des écosystèmes assez uniques. De même, il faudrait s'assurer qu'aucune espèce animale ne dépend strictement d'arbres vétérans pour les essences à grande longévité, par exemple, l'érable à sucre qui atteint 300 à 400 ans (Godman 1965).

\section{Le bois mort}

Les densités de chicots que nous avons calculées représentent une fraction seulement de la densité totale puisque les mesures se limitaient aux coniferes morts possédant un fin bout $<5 \mathrm{~cm}$ et aux feuillus portant encore presque toutes leurs branches. Nos chiffres ne permettent pas une comparaison directe avec d'autres études parce que les catégories de hauteur et de DHP varient énormément d'une à l'autre (Hély et al. 2000, Desponts et al. 2004, Roberge et Desrochers 2004, Park et al. 2005). La densité de chicots que nous avons estimée représente un minimum et concerne les chicots récemment apparus; elle a varié entre $\approx 20$ et 80 chicots/ha, et a eu tendance à demeurer plus faible dans les érablières que dans les forêts de conifères. La densité des gros chicots $(\mathrm{DHP}>20 \mathrm{~cm})$ était directement proportionnelle à celle de tous les chicots durant la période d'étude, ce qui suggère que l'abondance des gros chicots a eu tendance à évoluer en parallèle avec celle de l'ensemble des chicots entre les années 1970 et 1990. La densité de chicots que nous avons estimée représentait grossièrement entre $5 \%$ et $10 \%$ des arbres vivants présents dans les forêts du Québec. Dans la forêt boréale ontarienne, les chicots comptaient pour $12 \%$ à $18 \%$ des arbres selon la classe d'âge (Ferguson et Archibald 2002).

Durant la période d'étude, la densité des chicots a eu tendance à décroître uniquement dans l'érablière et la sapinière à bouleau jaune de l'ouest, alors que le contraire survint ailleurs dans les forêts de coniferes, avec des maxima notés dans les années 1980 ou 1990. Les chicots d'essences feuillues dominaient vraisemblablement dans les deux premiers sous-domaines, alors que ceux de coniferes le faisaient ailleurs.

La dernière épidémie de tordeuse des bourgeons de l'épinette a frappé les forêts québécoises entre les années 1970 et le début des années 1990 (Boulanger et Arseneault 2004). Cet insecte, qui peut tuer les épinettes et tout particulièrement le sapin baumier (Bouchard et al. 2005), a généré une quantité massive de chicots et de débris ligneux. Ceci s'explique facilement puisque le sapin baumier représente l'espèce d'arbres la plus répandue au Québec. Il domine dans les sapinières, mais il abonde également dans les autres domaines bioclimatiques (Fig. 7). D'ailleurs, l'indice d'activité de la tordeuse que nous avons utilisé expliquait $45 \%$ de la variation observée du nombre moyen de chicots dans les différents domaines au cours des trois décennies. Comme les chicots se renversent généralement en une ou deux décennies après la mort de l'arbre (Lee 1998, Garber et al. 2005), il faudra continuer à suivre l'évolution de la quantité de bois mort présent dans les forêts du Québec au cours des années à venir pour s'assurer d'une présence suffisante et continue. Durant la période d'étude, la faune tributaire du bois mort (Crête et al. 2004b) ne semble pas avoir vu ses habitats se raréfier substantiellement. 


\section{La composition et la diversité des forêts}

L'appréhension d'une augmentation de la proportion de feuillus qui excède celle des paysages naturels représente l'une des principales préoccupations actuelles face à la composition future des forêts québécoises aménagées (Grondin et al. 2003). Le remplacement du feu par la coupe a conduit notamment à un remplacement du pin blanc par le peuplier faux-tremble dans les forêts du Minnesota (Friedman et Reich 2005). Un remplacement massif des coniferes par les feuillus aurait un impact certain sur la faune forestière.

Nos résultats pour les arbres (Fig. 4) démontrent que la proportion de feuillus dans le paysage augmente clairement lorsque les peuplements de la pessière à mousses ont moins de 100 ans; le même phénomène semble se produire à un degré moindre dans les sapinières. Après une coupe, les feuillus de lumière peuvent proliférer parce qu'ils croissent plus rapidement que l'épinette noire et le sapin baumier. Les coniferes les rattrapent toutefois après quelques décennies. Laflèche et al. (2004) ont noté que le remplacement des feuillus par les coniferes, durant les 30 ans suivant une coupe, s'avérait cependant plus complet dans la pessière que la sapinière. Plus tard dans la vie du peuplement, la longévité différentielle des feuillus et des coniferes et le remplacement progressif des espèces expliquent pourquoi la proportion de feuillus tend à diminuer quand les peuplements deviennent âgés (Bergeron 2000). Le maintien d'une structure d'âge jeune dans les pessières et les sapinières aménagées conduit donc à une augmentation de la proportion de feuillus dans le paysage; toutefois, la situation ne devrait pas s'être modifiée substantiellement au cours des trois dernières décennies, sauf dans la sapinière à bouleau jaune de l'ouest, puisque la proportion de forêts âgées n'a pas varié beaucoup au Québec (Fig. 2). Même si la tendance n'était pas significative, la tordeuse des bourgeons de l'épinette a contribué à augmenter la proportion de feuillus durant la période d'étude, particulièrement dans la sapinière à bouleau blanc, en tuant sélectivement les coniferes (Fig. 4; Bouchard et al. 2005).

Chez les gaules (Fig. 5), la proportion de feuillus dans les différents domaines bioclimatiques était du même ordre de grandeur que chez les arbres durant les années 1970. Cependant, cette proportion augmenta substantiellement durant la période d'étude, ce qui pourrait signifier que les recrues qui remplaceront les arbres au cours des prochaines décennies accentueront la proportion de feuillus dans les forêts de coniferes du Québec. Nous ne croyons pas que ce soit le cas, car il s'agit d'un phénomène épisodique causé par la tordeuse des bourgeons de l'épinette, dont surtout les arbustes ont profité. Ruel et Huot (1993) ont noté que la tordeuse des bourgeons de l'épinette avait favorisé la régénération feuillue au détriment de celle des coniferes dans les sapinières du Québec. La tordeuse des bourgeons de l'épinette a provoqué une ouverture de la voûte forestière, ce qui a stimulé la croissance des gaules, comme en témoigne la relation positive qu'on a observée entre la fréquence des signes d'épidémie et la densité des gaules. Cependant, ce sont surtout les gaules feuillues qui ont profité de cette augmentation de l'énergie solaire en sous-bois, alors que les gaules et les semis de sapin en particulier subissaient de la mortalité causée par la tordeuse des bourgeons de l'épinette (Ruel et Huot 1993). Nous avons d'ailleurs observé une relation négative entre la proportion de sapin baumier chez les gaules et la proportion de feuillus.
Au niveau de l'Amérique du Nord, les forêts du sud du Québec s'avèrent relativement diversifiées en espèces d'arbres puisqu'on y compte près d'une centaine d'espèces contre 180 espèces dans les forêts les plus riches du sud-est du continent (Currie 1991). La diversité des arbres diminue cependant rapidement en progressant vers le nord du Québec (Currie 1991); aussi, est-il surprenant qu'on ait observé, durant les trois dernières décennies, la diversité la plus grande d'arbres dans les érablières à bouleau jaune. Ceci suggère que les peuplements forestiers aient été appauvris dans les domaines bioclimatiques plus au sud, vraisemblablement le résultat de l'activité humaine. Il se peut également que nos résultats soient un artefact lié à la taille uniforme des parcelles utilisées pour dénombrer les arbres et les gaules alors qu'elles pourraient devoir être plus grande en progressant du nord au sud pour capter la plus grande biodiversité végétale qui s'y trouve.

Contrairement à celle des arbres, la diversité des gaules a varié partout au cours de la période d'étude, sauf dans les deux domaines du sud. Le temps de réponse rapide des arbustes à la suite d'une perturbation explique vraisemblablement ce phénomène. En effet, nous avons observé une relation positive entre la densité des gaules et le taux de présence des signes d'épidémie ainsi qu'entre la densité des gaules et le nombre d'espèces présentes par placette. Ainsi, l'épidémie de tordeuse des bourgeons de l'épinette a ouvert la voûte forestière et a stimulé la croissance des arbustes et des arbrisseaux. Ce ne sont toutefois pas les espèces d'arbres feuillus susceptibles de remplacer les coniferes (bouleau à papier et peuplier faux-tremble) qui ont profité de cet accroissement de lumière en sous-bois, mais des espèces arbustives secondaires telles que le sorbier d'Amérique, l'érable à épis ou l'aulne rugueux. Ceci suggère qu'une épidémie de tordeuse des bourgeons de l'épinette provoque des changements de la composition végétale qui perdurent pendant quelques décennies (Bouchard et al. 2005). La diversité des gaules la plus grande s'observa dans les sapinières, alors qu'on devrait s'attendre à des sous-bois plus riches dans les érablières. L'accroissement des populations de cerfs de Virginie (Odocoileus virginianus), dans le sud du Québec, pourrait être partiellement responsable de la relative pauvreté des sous-bois puisqu'on a observé une relation négative entre la densité de ce cervidé et la densité de gaules (Boucher et al. 2004). Cette pauvreté peut également résulter de l'activité agricole, de l'usage historique des boisés et de leur isolement dans cette partie du Québec la plus densément peuplée. Contrairement à nos anticipations, la flore ligneuse du Québec ne s'est pas appauvrie durant la période d'étude et, au contraire, la tordeuse des bourgeons de l'épinette l'a enrichie temporairement. Conséquemment, la faune tributaire de certaines espèces ligneuses n'a pas vu ses ressources se raréfier entre les années 1970 et 1990.

\section{Conséquences pour la gestion}

Notre analyse illustre modestement la puissance du réseau des placettes permanentes mis en place au début des années 1970 pour suivre l'évolution des forêts québécoises. Nous avons utilisé quelques attributs du milieu forestier pour porter un jugement grossier sur l'évolution des habitats fauniques durant les trois dernières décennies. Conformément au concept du filtre brut, nous présumons que la faune forestière peut se maintenir et continuer à évoluer si nous 
conservons la gamme des écosystèmes forestiers présents dans les forêts sans aménagement. Cette hypothèse mérite cependant d'être testée en déterminant périodiquement l'aire de répartition et l'abondance relative des espèces animales forestières. Le réseau des parcelles permanentes s'enrichirait si l'on y accolait un programme de suivi de la faune forestière; comme les espèces évoluent individuellement et non en guilde ou groupe (Jablonski 1991), il faudrait suivre le plus grand nombre possible d'espèces pour s'assurer que l'aménagement forestier n'en met pas certaines en danger.

Sauf pour la pessière à mousses dont près de la moitié ne fait pas l'objet d'aménagement forestier, les peuplements âgés ont été très rares dans les forêts du Québec depuis au moins 30 ans. Il importe donc de soustraire un échantillon représentatif des derniers peuplements âgés de tout aménagement forestier dans les plus brefs délais. L'élargissement actuel du réseau des aires protégées du Québec et la création de refuges biologiques dans le cadre des nouveaux plans d'aménagement forestier constituent deux occasions d'atteindre cet objectif. On devrait porter une attention particulière aux forêts surannées, afin de déterminer si certaines espèces animales en sont totalement tributaires. De même, on devrait chercher à identifier les éléments de la flore ligneuse du sud du Québec vraisemblablement devenus rares, afin d'accentuer les efforts de protection et, au besoin, de mettre sur pied des programmes de restauration. On devrait également tenter de déterminer si certains éléments de la faune pourraient souffrir de la diversité ligneuse diminuée des forêts du sud du Québec.

L'acceptation sociale de l'aménagement forestier signifie, au Québec, un glissement vers une pyramide d'âge plus jeune des peuplements et des arbres. Ainsi, les forêts aménagées seront davantage favorables à l'orignal et moins au caribou forestier, par exemple. Les gestionnaires de la faune ont le mandat premier de conserver les espèces et leurs habitats, ainsi que de maintenir la connectivité entre les écosystèmes, afin que la sélection naturelle continue à jouer son rôle. Ils possèdent divers outils pour atteindre ce but, notamment le réseau des aires protégées, la protection des espèces, le contrôle de l'exploitation et l'aménagement forestier. Même si l'on tente de conserver les attributs du milieu forestier dans les forêts aménagées, les gestionnaires de la faune ont toutefois besoin d'inventaires périodiques d'animaux pour s'assurer qu'ils remplissent bien leur mandat.

\section{Remerciements}

Nous remercions G. Blais, F. Labbé et P. Morin qui nous ont aimablement fourni des informations sur la base de données des placettes permanentes ainsi que G. Daigle qui nous a prodigué des conseils pour l'analyse statistique. Nous remercions également J. Berthiaume qui a préparé les figures ainsi que M. Auger, L. Deschamps et J. Fortin pour les données d'inventaires sur l'épidémie de tordeuse du bourgeon de l'épinette. Les commentaires constructifs de G. Blais, M. Darveau, S. Gauthier, P. Grondin et R. Lafond nous ont permis d'améliorer ce manuscrit. Le ministère des Ressources naturelles et de la Faune du Québec a rendu cette analyse possible.

\section{Références}

Anonyme. 1972. Relations des Jésuites 1611-1672. Six volumes. Édition du Jour, Montréal, Qc.

Anonyme 2001a. Normes d'inventaire forestier - placetteséchantillons permanentes. Ministère des Ressources naturelles, Québec, Qc.

Anonyme 2001b. Stratégie québécoise sur la diversité biologique 2002-2007. Gouvernement du Québec, Québec, Qc.

Anonyme 2003. Manuel d'aménagement forestier, $4^{\mathrm{e}}$ édition. Ministères des Ressources naturelles, de la Faune et de Parcs, publ. 2003-3063.

Anonyme 2004. Rapport préliminaire sur l'évolution de la forêt du Québec méridional des années 1970 aux années 1990. Ministère des Ressources naturelles, de la Faune et des Parcs, préparé pour la Commission d'étude scientifique, technique, publique et indépendante chargée d'examiner la gestion des forêts publiques du domaine de l'État.

Barrette, Y., G. Gauthier et A. Paquette. 1996. Aménagement de la forêt pour fins de production ligneuse. Dans Manuel de foresterie. pp. 648-672. Les Presses de l'université Laval, Québec, Qc.

Bergeron, Y. 2000. Species and stand dynamics in the mixed woods of Québec's southern boreal forest. Ecology 81: 1500-1516.

Bergeron, Y. 2004. Is regulated even-aged management the right strategy for the Canadian boreal forest? For. Chron. 80: 458-462.

Bergeron, Y., S. Gauthier, V. Kafka, P. Lefort et D. Lesieur. 2001. Natural fire frequency for the eastern Canadian boreal forest: consequences for sustainable forestry. Can. J. For. Res. 31: 384-391.

Bergerud, A. T. et J. P. Elliot. 1986. Dynamics of caribou and wolves in northern British Columbia. Can. J. Zool. 64: 1515-1529.

Bouchard, M., D. Kneeshaw et Y. Bergeron. 2005. Mortality and stand renewal patterns following the last spruce budworm outbreak in mixed forests of western Québec. For. Ecol. Manage. 204: 297-313. Boucher, S., M. Crête, J.-P. Ouellet, C. Daigle et L. Lesage. 2004. Large-scale trophic interactions: white-tailed deer growth and forest understory. Écoscience 11: 286-295.

Boulanger, Y. et D. Arseneault. 2004. Spruce budworm outbreaks in eastern Québec over the last 450 years. Can. J. For. Res. 34: 1035-1043.

Bradshaw, C.J.A., S. Boutin et D.M. Hebert. 1997. Effects of petroleum exploration on woodland caribou in northeastern Alberta. J. Wildl. Manage. 61: 1127-1133.

Courtois, R. 2003. La conservation du caribou forestier dans un contexte de perte d'habitat et de fragmentation du milieu. Thèse de doctorat, Univ. Québec à Rimouski, Rimouski, Qc.

Courtois, R., J.-P. Ouellet, A. Gingras, C. Dussault, L. Breton et J. Maltais. 2003. Historical changes and current distribution of caribou, Rangifer tarandus, in Québec. Can. Field-Nat. 117: 399-414. Courtois, R., J.-P. Ouellet, C. Dussault et A. Gingras. 2004. Forest management guidelines for forest-dwelling caribou in Québec. For. Chron. 80: 598-607.

Crête, M., D. Baril, C. Langlois et R. Daigle. 2004a. Prise en compte des préoccupations fauniques lors de la planification de l'aménagement forestier : analyse pour deux zones d'exploitation contrôlée. Ministère des Ressources naturelles, de la Faune et des Parcs, publ. 8115-04-09.

Crête, M., S. Brais, M. Campagna, M. Darveau, M. Desponts, S. Déry, P. Drapeau, B. Drolet, J.-P. Jetté, C. Maisonneuve, A. Nappi et P. Petitclerc. 2004b. Pourquoi et comment maintenir du bois mort dans les forêts aménagées du Québec. Société de la faune et des parcs du Québec, publ. \# 8108-04-06.

Currie, D.J. 1991. Energy and large-scale patterns of animal- and plant- species richness. Am. Nat. 137: 27-49.

Desponts, M., G. Brunet, L. Bélanger et M. Bouchard. 2004. The eastern boreal old-growth balsam forest: a distinct ecosystem. Can. J. Bot. 82: 830-849. 
Drapeau, P., A. Leduc, Y. Bergeron, S. Gauthier et J.-P. Savard. 2003. Les communautés d'oiseaux des vieilles forêts de la pessière à mousses de la ceinture d'argile : problèmes et solutions face à l'aménagement forestier. For. Chron. 79 : 531-540.

Dussault, C., R. Courtois, J. Huot et J.-P. Ouellet. 2001. The use of forest maps for the description of wildlife habitats: limits and recommendations. Can. J. For. Res. 31: 1227-1234.

Dyer, S.J., J. P. O’Neil, S.M. Wasel et S. Boutin. 2001. Avoidance of industrial development by woodland caribou. J. Wildl. Manage. 65: 531-542.

Dyer, S.J., J.P. O’Neil, S.M. Wasel et S. Boutin. 2002. Quantifying barrier effects of roads and seismic lines on movements of female woodland caribou in northeastern Alberta. Can. J. Zool. 80: 839-845.

Ferguson, S. H. et D. J. Archibald. 2002. The 3/4 power law in forest management: how to grow dead trees. For. Ecol. Manage. 169: 283-292.

Friedman, S.K. et P.B. Reich. 2005. Regional legacies of logging: departure from presettlement forest conditions in northern Minnesota. Ecol. Appl. 15: 726-744.

Garber, S.M., J.P. Brown, D.S. Wilson, D.A. Maguire et L.S. Heath. 2005. Snag longevity under alternative silvicultural regimes in mixed-species forests of central Maine. Can. J. For. Res. 35: 787-796. Godman, R.M. 1965. Sugar maple (Acer saccharum Marsh.). Dans H. A. Fowells (comp.). Silvics of forest trees of the United States. P. 6tk73. U.S. Department of Agriculture, Agriculture Handbook 271. Washington D.C.

Grondin, P., L. Bélanger, V. Roy, J. Noël et A. Cimon. 2003. Envahissement des parterres de coupe par les feuillus de lumière (enfeuillement). Dans P. Grondin et A. Cimon (coord.). Les enjeux de biodiversité relatifs à la composition forestière. pp. 131-174. Ministère des Ressources naturelles, de la faune et des Parcs, publ. 2003-3070.

Hély, C., Y. Bergeron et M. D. Flannigan. 2000. Coarse woody debris in the southeastern Canadian boreal forest: composition and load variations in relation to stand replacement. Can. J. For. Res. 30: 674-687.

Harper, K.A., Y. Bergeron, P. Drapeau, S. Gauthier et L. De Grandpré. 2005. Structural development following fire in black spruce boreal forest. For. Ecol. Manage. 206: 293-306.

Hunter, M. L. Jr. 1999. Maintaining biodiversity in forest ecosystems. Cambridge University Press, Cambridge, UK.

Imbeau, L., M. Mönkkönen et A. Desrochers. 2001. Long-term effects of forestry on birds of the eastern Canadian boreal forests: a comparison with Fennoscandia. Cons. Biol. 15: 1151-1162.

Jablonski, D. 1991. Extinctions: a paleontological perspective. Science 253: 754-757

Krankina, O.N., M.E. Harmon, Y.A. Kukuev, R.F. Treyfeld, N.N. Kashpor, V.G. Kresnov, V.M. Skudin, N.A. Portoasov, M. Yatskov, G. Spycher et W.D. Povarov. 2002. Coarse woody debris in forest regions of Russia. Can. J. For. Res. 32: 768-778.

Laflèche, V., J. Bégin et J.-C. Ruel. 2004. Effets de la coupe avec protection de la régénération sur la production des peuplements forestiers. Québec, Min. Ressources naturelles, Faune et Parcs, Mémoire de recherche forestière \# 146.

Lee, P. 1998. Dynamics of snags in aspen-dominated midboreal forests. For. Ecol. Manage. 105: 263-272.

Lesage, L., M. Crête, J. Huot et J.-P. Ouellet. 2002. Use of forest maps versus field surveys to measure summer habitat selection and sexual segregation in northern white-tailed deer. Can. J. Zool. 80: 717-726. Mahoney, S.P. et J.A. Schaefer. 2002. Hydroelectric development and the disruption of migration in caribou. Biol. Cons. 107: 147-153.
Mahoney, S.P. et J.A. Virgl. 2003. Habitat selection and demography of a nonmigratory woodland caribou population in Newfoundland. Can. J. Zool. 81: 321-334.

Maier, J.A.K., S.M. Murphy, R.G. White et M.D. Smith. 1998. Responses of caribou to overflights by low-altitude jet aircraft. J. Wildl. Manage. 62: 752-766.

Nelleman, C. et R.D.Cameron. 1996. Effects of pretroleum development on terrain preferences of calving caribou. Arctic 49: 23-28.

Nelleman, C., P. Jordhøy, O.-G. Støen et O. Strand. 2000. Cumulative impacts of tourist resorts on wild reindeer (Rangifer tarandus tarandus) during winter. Arctic 53: 9-17.

Nelleman, C., I. Vistnes, P. Jordhøy et O. Strand. 2001. Winter distribution of wild reindeer in relation to power lines, roads and resorts. Biol. Cons. 101: 351-360.

Parent, B. 1991. Ressource et industrie forestières. Ministère des Forêts, publ. 91-3009.

Parent, B. et C. Fortin. 2003. Ressources et industries forestières, portrait statistique édition 2003. Ministère des Ressources naturelles, de la Faune et des Parcs, publ. 2003-3123.

Park, A., D. Kneeshaw, Y. Bergeron et A. Leduc. 2005. Spatial relationships and tree species associations across a 236-year boreal mixewood. Can. J. For. Res. 35: 750-761.

Poole, K.G., A.D. Porter, A. de Vries, C. Maundrell, S.D. Grindal et C. Cassady St. Clair. 2004. Suitability of a young deciduousdominated forest for American marten and the effects of forest removal. Can. J. Zool. 82: 423-435.

Potvin, F., L. Bélanger et K. Lowell. 1999. Validité de la carte écoforestière pour décrire les habitats fauniques à l'échelle locale : une étude de cas en Abitibi-Témiscamingue. For. Chron. 75 : 851-859.

Potvin, F., L. Bélanger et K. Lowell. 2000. Marten habitat selection in a clearcut boreal landscape. Cons. Biol. 14 : 844-857.

Reimers, E., S. Efestøl et J.E. Colman. 2003. Behavior responses of wild reindeer to direct provocation by a snowmobile or skier. J. Wildl. Manage. 67: 747-754.

Roberge, J.-M. et A. Desrochers. 2004. Comparaison des caractéristiques des gros chicots entre la sapinière primitive et une sapinière aménagée sur la péninsule gaspésienne, Québec. Can. J. For. Res. $34: 2382-2386$.

Ruel, J.-C. et M. Huot. 1993. Impact de la tordeuse des bourgeons de l'épinette [Choristoneura fumiferana (Clem.)] sur la régénération des sapinières après coupe à blanc. For. Chron. 69: 163-172.

SAS Institute. 1999. SAS/STAT user's guide. Release 8.02. SAS Insitute Inc. Cary, North Carolina.

Saucier, J.-P., P. Grondin, A. Robitaille et J.-F. Bergeron. 1998. Les zones de végétation et les domaines bioclimatiques du Québec. Ministère des Ressources naturelles, Carte couleur.

Schaefer, J. A. 2003. Long-term range recession and the persistence of caribou in the taiga. Cons. Biol. 17: 1435-1439.

Seip, D.R. 1992. Factors limiting woodland caribou populations and their interrelationships with wolves and moose in southeastern British Columbia. Can. J. Zool. 70: 1494-1503.

Simard, H. et A. Bouchard. 1996. The precolonial $19^{\text {th }}$ century forest of the Upper St. Lawrence region of Québec: a record of its exploitation and transformation through notary deeds of wood sales. Can. J. For. Res. 26: 1670-1676.

Thompson, I.D. 1991. Could marten become the spotted owl of eastern Canada? For. Chron. 67: 136-140. 